\title{
Spillover effects and the evolution of firm clusters
}

\author{
G.-I. Bischi ${ }^{\text {a }}$, H. Dawid ${ }^{\text {b }}$, M. Kopel ${ }^{\mathrm{c}, *}$ \\ a Instituto di Scienze Economiche, University of Urbino, Urbino, Italy \\ ${ }^{\mathrm{b}}$ Department of Economics, University of Southern California, Los Angeles, CA, USA \\ ${ }^{c}$ Department of Managerial Economics and Industrial Organization, \\ Vienna University of Technology, Vienna, Austria
}

Received 4 May 2001; received in revised form 4 May 2001; accepted 9 August 2001

\begin{abstract}
In this paper we consider the impact of spillovers occurring within each of two groups of firms on the long run agglomeration patterns in a market. In each period every single firm can either produce for this market or choose some outside option (e.g. a risky asset). Firms switch between the two options based on information about the relative profitability of the market and the outside option. In the market, due to spillovers, the production costs are influenced by the number of firms from the same population which are in the market. The resulting model describes the evolution of the size of the two firm clusters and their market shares over time. We provide a global analysis of the existence and basins of attraction of equilibria to address the question what impact different constellations of spillover effects have on the growth of dominant respectively incoming clusters. We demonstrate that the basins of attraction of coexisting long run equilibria do not depend continuously on the size of the spillover effects. Furthermore, an increase in the initial cluster size is not necessarily beneficial if the switching behavior of firms is fast.
\end{abstract}

(C) 2003 Elsevier Science B.V. All rights reserved.

JEL classification: D62; D83; L52

Keywords: Spillover effects; Evolutionary dynamics; Market entry and exit; Equilibrium selection

\section{Introduction}

The clustering of firms related to an industry in relatively small geographical regions is a well-observed phenomenon. Industries where such agglomeration patterns have been observed are, e.g. car manufacturing, computer manufacturing, fashion design or the chemical industry; see Audretsch and Feldman (1996) and, in particular, Ellison and Glaeser (1997) for an extensive analysis of the geographic concentration of several different US

\footnotetext{
* Corresponding author. Fax: +43-1-58801-33096.

E-mail address: kopel@mail.ibab.tuwien.ac.at (M. Kopel).
} 
industries. Among the main reasons for such clustering are cost reducing externalities due to technological and intellectual spillovers from other similar companies in the region. The size of these externalities may differ between regions and industries, as empirical studies show. For example, the ability of Japanese car manufacturers to yield a sustainable market share on western markets is generally attributed to their keiretsu organization, which facilitates internal spillovers, and their superior ability for technological sourcing, which allows them to use externally-based technologies much faster and more efficient than their US competitors (Mansfield, 1988).

It is crucial for the success of a cluster in the long run that joining the cluster becomes attractive for local entrepreneurs and investors. The evolution of the size of the cluster is a decentralized process governed by production decisions of numerous individual firms. This raises the question whether general factors can be identified which facilitate or hinder the long run development of a cluster. In particular, we are interested how initial cluster size and ability to utilize spillover effects influences the competitive position and the growth potential of a firm cluster in the market. Which differences in the size of spillover effects allow an initially marginal cluster to coexist with another cluster of firms in the market? Under which circumstances can larger spillover effects lead to a market takeover by one cluster? Questions like that are particularly relevant for regional planners who are interested in attracting or building up a certain industry cluster in their region by providing appropriate infrastructure and incentives for the emergence of spillovers and by attracting initial investments. A few authors have provided policy recommendations on these issues based on empirical studies (Chuang and Lin, 1999; Porter, 1998) or static models (Carlisle, 1992). However, they did not explicitly take into account the interaction of distinct clusters in the market and the dynamics of industry evolution.

In this paper we carry out a dynamic evolutionary analysis of the competition of two industry groups in a market where spillover effects are present. Our focus here is on industries like the software industry with low barriers of entry and frequent exit and entry of firms. The size of the two industry groups depends on the attractivity of the market for both groups, which is expressed by the profits achieved by firms who are producing for the market in relation to the profit of some outside option. The market entry and exit decision of firms is made on the basis of information about the relative profitability of the market which has been collected via direct communication within the group. We analyze the evolution of the size and market shares respectively for both firm clusters. Using this evolutionary approach we will address the questions of industrial evolution stated earlier which have, to our best knowledge, not been analyzed in the framework of a dynamic model. Entry and exit dynamics in the presence of externalities have sporadically been analyzed to address questions of firm organization (Friedman and Fung, 1996) and location and education choice in cities (Benabou, 1993).

Dynamic evolutionary models based on local interaction of boundedly rational agents have been used with increasing frequency to gain a better understanding of numerous important economic problems. ${ }^{1}$ Whereas, this type of research initially focused on the

\footnotetext{
1 The fields of application include bargaining models (Young, 1993; Ellingsen, 1997), auctions (Dawid, 1999a; $\mathrm{Lu}$ and McAfee, 1996), the formation of social norms (Young, 1998) or various models of market interaction (Qin and Stuart, 1997; Vega-Redondo, 1997).
} 
notion of evolutionary stability (ESS) and deterministic evolutionary dynamics, the larger part of recent contributions has used stochastic models including 'experimentation' or 'error' terms. The concept of stochastic stability (Ellison, 2000) which is usually applied in this framework has the appealing feature that it generates, for most of the models, a unique long run prediction and, therefore, is a useful tool for equilibrium selection based on evolutionary arguments. However, it has to be pointed out that these predictions often hold only in the very long run and there may be a large probability that the process stays for a very long time close to some state different from the stochastically stable one. ${ }^{2}$ Whereas, it is reasonable to consider ultra long run outcomes of processes which govern, e.g. the emergence of social norms, for the analysis of industry evolution a shorter time-horizon is suitable. For example, if we consider an emerging industry the initial evolution towards a stable structure most likely determines the appearance of this industry for its entire life cycle. Transitions to different structures which are triggered entirely by numerous simultaneous implementation errors or experiments of firms seem to be hardly relevant for the understanding of such an evolution. Accordingly, here we look at the intermediate run using a deterministic model of the evolution of the size of the two industry groups. We follow Friedman (1998), who points out that "[I]n most applied work it suffices to identify the evolutionary equilibria and their basins of attraction", (Friedman, 1991, p. 639), and characterize the equilibria in our model and their basins depending on the relative size of the spillover effects. To this end we employ the theory of critical curves and demonstrate how this concept can be used to understand and predict the changes of the basins of attraction in highly non-linear evolutionary models with multiple coexisting steady states. Although this technique has been sporadically applied in economic modeling in the past (Bischi et al., 2000; Bischi and Kopel, 2001), this is the first study where the merits of this approach for evolutionary analysis are explored.

The paper is organized as follows. In Section 2 we introduce the model. Section 3 characterizes all the possible constellations of fixed points of the model and some general results about local stability. Section 4 then studies the long run properties of the model when the size of spillover effects are symmetric between the two populations. Section 5 deals with the case where spillover effects are asymmetric and Section 6 examines the effect of the speed of market exit and entry on the evolution of the industry. We summarize and discuss our main findings in Section 7. All proofs are given in the Appendix A.

\section{Markets and spillovers}

Consider two groups of firms $i=1,2$ which have to decide whether to produce for a certain market or not. Alternatively, you might think of two groups of investors who have to decide whether to invest in a certain local industry branch or not. As laid out in the introduction, the assignment into one of the groups may be due to several reasons like the location or the national origin of the firms. The only crucial observation here is that there is more flow of information within a group than between the two groups. To keep matters as

\footnotetext{
${ }^{2}$ For example Ellison (2000), points out that “... it is inherently limited in scope to a characterization of the very long run limit. This can be problematic because evolution in these models is at times so slow as to be of limited practical importance."
} 
simple as possible we assume that the two groups are of the same size and denote by $x_{i t}$ the fraction of firms in population $i=1,2$, which are in the market at time $t$. This fraction might as well be interpreted as the fraction of available capital in each country which is invested in firms in the market. Assuming constant returns to scale, it does not make a difference for any of our arguments whether output within one country is produced by several large or many small firms as long as no firm has relevant market power.

The focus in this paper is on market exit and entry behavior of firms and no explicit model of the output decision of the firms is provided (see Ghemawat and Spence, 1985 for an analysis of the implications of spillover effects on the firms production decision). For reasons of simplicity it is assumed that every firm in the market produces 1 unit of a homogeneous good per period. Aggregate output in the market is then given by $\left(x_{1}+x_{2}\right)$ times the number of firms. The market clearing price is determined by an inverse demand function

$$
p=p\left(x_{1}+x_{2}\right) .
$$

Due to spillovers within each group, marginal costs are smaller the more other firms in the population produce the same good. We include such cost externalities in our model by assuming that the unit costs of a firm depend on the number of firms from the own populations which produce the good. Hence, we express unit costs of a firm in population $i$ by $c_{i}\left(x_{i}\right)$, where $\partial c_{i} / \partial x_{i} \leq 0, i=1,2$. Furthermore, in line with previous theoretical work on the effects of spillovers, we assume that unit costs decline with a decreasing rate (Kamien et al., 1992): $\partial^{2} c_{i} / \partial x_{i}^{2}>0, i=1,2$. This gives a per period profit of

$$
\pi_{i}\left(x_{1}, x_{2}\right)=p\left(x_{1}+x_{2}\right)-c_{i}\left(x_{i}\right) .
$$

The profit of a firm which stays out of the market (i.e. chooses the outside option) is modeled as a stochastic variable. Outside profit of firm $f$ in population $i$ at time $t$ is $u_{f, t}^{i}$ with expected value $U_{i}$. Outside profits are independent across individual firms in a population and time. We write $u_{f, t}^{i}=U_{i}+\epsilon_{f, t}^{i}$ where the density of $\epsilon_{f, t}^{i}$ is independent of $f$ and $t$, has full support $\mathbb{R}$ and is unimodal and symmetric with respect to 0 . The distribution function of $\epsilon_{f, t}^{i}$ is denoted by $\Theta_{i}$.

Each period $t=0, \ldots, \infty$ every firm decides whether to enter or to exit the market. If a firm samples another firm (in the same population) which has chosen a different action in the previous period, it switches (i.e. exits or enters) whenever the profit of the other firm has been larger than its own profit. The sampling procedure is assumed to be uniform and stochastically independent from the outside profit $u_{f, t}^{i}$. The more firms are outside the market the more likely it is for an incumbent to sample such an 'outsider' and to get information about the outside option. The probability that an arbitrary firm in population $i$ which is now in the market exits after period $t$ is given by:

$$
\begin{aligned}
p_{\text {out }}^{i}\left(x_{1}, x_{2}\right) & =\left(1-x_{i}\right) \mathbb{P}\left(\pi_{i}\left(x_{1}, x_{2}\right)<U_{i}+\epsilon_{f, t}^{i}\right) \\
& =\left(1-x_{i}\right)\left(1-\Theta_{i}\left(\pi_{i}\left(x_{1}, x_{2}\right)-U_{i}\right)\right) .
\end{aligned}
$$

On the other hand, a firm currently outside the market enters the market with probability

$$
p_{\text {in }}^{i}\left(x_{1}, x_{2}\right)=x_{i} \mathbb{P}\left(\pi_{i}\left(x_{1}, x_{2}\right)>U_{i}+\epsilon_{f, t}^{i}\right)=x_{i} \Theta_{i}\left(\pi_{i}\left(x_{1}, x_{2}\right)-U_{i}\right)
$$


The expected fraction of population $i$ firms $(i=1,2)$ in the market is therefore:

$$
\begin{aligned}
x_{i, t+1} & =x_{i, t}+\left(1-x_{i, t}\right) p_{\text {in }}^{i}\left(x_{1, t}, x_{2, t}\right)-x_{i, t} p_{\text {out }}^{i}\left(x_{1, t} x_{2, t}\right) \\
& =x_{i, t}+x_{i, t}\left(1-x_{i, t}\right)\left(\Theta_{i}\left(\pi_{i}\left(x_{1, t}, x_{2, t}\right)-U_{i}\right)-\left(1-\Theta\left(\pi_{i}\left(x_{1, t}, x_{2, t}\right)-U_{i}\right)\right)\right. \\
& =x_{i, t}+x_{i, t}\left(1-x_{i, t}\right) G_{i}\left(\pi_{i}\left(x_{1, t}, x_{2, t}\right)-U_{i}\right),
\end{aligned}
$$

where $G_{i}(x):=2 \Theta_{i}(x)-1$. The evolution of the fractions $x_{1}$ and $x_{2}$ are hence described by a nonlinear deterministic system in discrete time. Word of mouth dynamics similar to this one have been analyzed by Ellison and Fudenberg $(1993,1995)$ or Dawid (1999b). It is obvious that the shape of the function $G_{i}$ depends on the distribution function $\Theta_{i}$. However, from the fact that $\Theta_{i}$ is a distribution function and the properties of the corresponding density (uni-modality and symmetry) it is easy to derive the following statements for $i=1,2$ :

$$
G_{i}(0)=0, \quad \lim _{x \rightarrow \infty} G_{i}(x)=1, \quad \lim _{x \rightarrow-\infty} G_{i}(x)=-1 .
$$

Furthermore, $G_{i}(x)$ is symmetric with respect to 0 , convex on $(-\infty, 0]$ and concave on $[0, \infty)$. The slope of $G_{i}$ at 0 is twice the altitude of the hump of the unimodal density. It will turn out that the qualitative properties of the long run behavior of the dynamics in many cases crucially depend on the 'speed' of the flow towards the action with the higher expected profit. We will use $G_{i}^{\prime}(0)$ as a measure of this speed and denote it by $\lambda_{i}{ }^{3}$

Studying the nonlinear two-dimensional dynamical system (1) allows us to derive qualitative features of the evolution of the fraction of firms of the two populations which are in the considered market. In particular, we are interested in the question how initial market shares of firms of the two populations, $x_{1,0}$ and $x_{2,0}$, and differences in spillovers influence the convergence properties of the evolutionary process to some long run equilibrium (the agglomeration pattern). To answer this question, we will provide an extensive analysis of the equilibria and their basins of attraction. However, before we proceed we need to be more specific about the functions involved.

We will assume that the demand curve is linear

$$
p\left(x_{1}+x_{2}\right)=P_{0}-B\left(x_{1}+x_{2}\right) .
$$

Furthermore, we use the following rational expression for the unit costs of a firm in population $i$ :

$$
c_{i}\left(x_{i}\right)=\frac{C_{i}}{1+\beta_{i} x_{i}}, \quad i=1,2 .
$$

The parameter $\beta_{i}>0$ incorporates the effect of spillovers within a firm cluster. As explained earlier, the qualitative properties of the cost function are inspired by existing theoretical and empirical work. The profit of a firm in population $i$ which is in the market is then given by

$$
\pi_{i}\left(x_{1}, x_{2}\right)=P_{0}-B\left(x_{1}+x_{2}\right)-\frac{C_{i}}{1+\beta_{i} x_{i}}, \quad i=1,2 .
$$

\footnotetext{
${ }^{3}$ For many classes of distribution functions, like the normal distribution, a large slope of $G_{i}$ at 0 corresponds to a small variance of the outside profit; for example for the normal distribution $\lambda_{i}$ is inversely proportional to $\sigma$.
} 
We will always assume that if all firms from both populations are in the market, the payoff for firms in the market is smaller than the expected outside profit. This assumption rules out the rather unrealistic and uninteresting case where the market is so much more attractive than the outside option that all firms from both populations want to enter or stay under all circumstances. On the other hand, the monopoly profit of the firm entering the market should be larger than the expected outside profit. These conditions are represented by the following inequalities:

$$
\begin{array}{ll}
P_{0}-2 B-\frac{C_{i}}{1+\beta_{i}}<U_{i}, & i=1,2 \\
P_{0}-C_{i}>U_{i}, & i=1,2 .
\end{array}
$$

Additionally, we make the more technical assumption that, if there were no spillovers, the expected profit of the outside option would be higher than the profit in the market if half of the firms are in the market

$$
P_{0}-B-C_{i}<U_{i}, \quad i=1,2 .
$$

One of the main points we will make in this paper is to show that in the presence of large spillovers a population of firms can invade a market despite the fact that their initial market share is small. Accordingly, it is sensible to assume that these spillovers are the reason why the market may become, on average, more attractive than the outside option. By making this assumption we avoid the discussion of several cases. However, it would be straightforward to extend the analysis to cases where this assumption does not hold.

Using the expressions given earlier, we obtain the following evolutionary model which describes the dynamics of the fraction of firms from both populations in the market:

$$
\begin{aligned}
& x_{1, t+1}=x_{1, t}+x_{1, t}\left(1-x_{1, t}\right) G_{1}\left(A_{1}-B\left(x_{1, t}+x_{2, t}\right)-\frac{C_{1}}{1+\beta_{1} x_{1}}\right) \\
& x_{2, t+1}=x_{2, t}+x_{2, t}\left(1-x_{2, t}\right) G_{2}\left(A_{2}-B\left(x_{1, t}+x_{2, t}\right)-\frac{C_{2}}{1+\beta_{2} x_{2}}\right),
\end{aligned}
$$

where $A_{i}:=P_{0}-U_{i}$. We define $T:[0,1]^{2} \mapsto[0,1]^{2}$ as the right hand side of (5) and using this notation the system reads $x_{t+1}=T\left(x_{t}\right)$. In general terms we have derived a two-population evolutionary model with non-linear payoff functions and inter- and intra-population interaction.

\section{Fixed points and local stability}

Obviously, the state space $\mathcal{S}:=[0,1] \times[0,1]$ is invariant under the dynamics (5) and all four corners are fixed points. A standard local stability analysis further shows that under assumptions ( 3$)$ the two corners $(0,0)$ and $(1,1)$ are unstable. ${ }^{4}$ The corner $(1,0)$ is locally

\footnotetext{
${ }^{4}$ The details of the local stability analysis can be found in a technical appendix available upon request from the authors.
} 
asymptotically stable if,

$$
\begin{aligned}
& P_{0}-B-\frac{C_{1}}{1+\beta_{1}}>U_{1}, \\
& P_{0}-B-C_{2}<U_{2},
\end{aligned}
$$

and $(0,1)$ is locally asymptotically stable under the symmetric conditions

$$
\begin{aligned}
& P_{0}-B-C_{1}<U_{1}, \\
& P_{0}-B-\frac{C_{2}}{1+\beta_{2}}>U_{2} .
\end{aligned}
$$

Besides these four fixed points, the system may also have additional stationary points. Every point in the interior of $\mathcal{S}$ where $\pi_{1}\left(x_{1}, x_{2}\right)=U_{1}$ and $\pi_{2}\left(x_{1}, x_{2}\right)=U_{2}$ is a fixed point of (5). Furthermore, fixed points exist on the upper and lower boundary of $\mathcal{S}$ where $\pi_{1}\left(x_{1}, x_{2}\right)=U_{1}$ and on the left and right boundary where $\pi_{2}\left(x_{1}, x_{2}\right)=U_{2}$. To facilitate the analysis, we define the curves $F_{i}, i=1,2$ as the set of all points $\left(x_{1}, x_{2}\right)$ where the profit in the market equals the expected outside profit for a firm of population $i$, i.e.

$$
F_{i}=\left\{\left(x_{1}, x_{2}\right) \in[0,1]^{2} \mid \pi_{i}\left(x_{1}, x_{2}\right)=U_{i}\right\} .
$$

Interior equilibria exist at all intersections of the curves $F_{1}$ and $F_{2}$. Fixed points at the boundary occur either at the intersection of $F_{1}$ with $x_{2}=0$ or 1 , or at the intersection of $F_{2}$ with $x_{1}=0$ or 1 . Note however, that fixed points on the boundary might not correspond to Nash equilibria of the model. In the following proposition we characterize the fixed points (other than the vertices) of the dynamics under our assumptions.

Proposition 1. For $\beta_{i}>0$ the dynamical system (5) can have at most one fixed point in the interior of $[0,1]^{2}$ and if it exists it is always unstable. Additionally, there can be either at most two fixed points on the boundary $x_{1}=1$ or one fixed point on $x_{1}=0$ and either at most two fixed points on the boundary $x_{2}=1$ or one fixed point on $x_{2}=0$. There can never be fixed points on $x_{i}=0$ and $1, i=1,2$ simultaneously for the same values of the parameters.

Taking into account Proposition 1 we conclude that our model can have up to nine coexisting fixed points. There are always four at the vertices of $[0,1]^{2}$, at most four on the boundary and at most one in the interior of the unit square. Short introspection further establishes that there can be at most one stable equilibrium in the interior of a boundary line of the unit square. Therefore, all together there can be at most four locally stable fixed points. Any of these stable fixed points is the potential long run outcome of the evolutionary process driven by the switching behavior of the firms. Standard arguments used in the evolutionary game theory literature (Weibull, 1995) imply that every locally stable fixed point corresponds to a Nash equilibrium of the underlying two population game. Which locally stable equilibrium is actually chosen depends on the initial market share firms in population $i$ have. In order to obtain a thorough understanding of the interplay between initial market shares, long run outcomes and their dependence on parameters, for each equilibrium we need a characterization of the set of initial conditions for which the process 
converges to it. In other words, what is needed is not only an identification of the set of locally stable fixed points, but also a characterization of their basins of attraction and the changes these basins undergo as parameters are varied.

To make matters simple, we introduce a coherent notation for all fixed points on the boundary which we will use throughout the analysis. We denote the vertices as: $0=(0,0), V_{\mathrm{I}}=$ $(1,0), V_{\mathrm{II}}=(1,1), V_{\mathrm{III}}=(0,1)$, and the unique interior fixed point as $S=\left(s_{1}, s_{2}\right)$. Obviously, $V_{\mathrm{I}}$ characterizes an agglomeration pattern where all firms of population 1 are in the market and all firms of population 2 choose the outside option. Conversely, in $V_{\text {III popula- }}$ tion 2 firms are in the market and population 1 firms choose the outside option. We denote by $P_{\mathrm{I}}=\left(p_{\mathrm{I} 1}, 0\right)$ the interior fixed point on the boundary line $x_{2}=0$, by $Q_{\mathrm{II}}=\left(1, q_{\mathrm{II} 2}\right)$ and $P_{\mathrm{II}}=\left(1, p_{\mathrm{II} 2}\right)$ the two fixed points on $x_{1}=1$, where $q_{\mathrm{II} 2}<p_{\mathrm{II} 2}$, by $Q_{\mathrm{III}}=\left(q_{\mathrm{III} 1}, 1\right)$ and $P_{\mathrm{III}}=\left(p_{\mathrm{III} 1}, 1\right)$ the two fixed points on $x_{2}=1$, where $q_{\mathrm{III} 1}<p_{\mathrm{III} 1}$ and by $P_{\mathrm{IV}}=\left(0, p_{\mathrm{IV} 2}\right)$ the interior fixed point on $x_{1}=0$. For example, $P_{\mathrm{III}}$ characterizes a situation where in the long run firms of population 2 dominate the market. However, not all firms of population 1 are driven out of the market: a fraction of firms of population 1 coexists.

To keep our exposition as clear and simple as possible we assume that the constant unit costs of a single firm from either population are identical: $C_{1}=C_{2}=C$. In other words, the profit a single firm can achieve when entering a market where no other firm of the same population is in, is independent of the population the firm belongs to. Furthermore, we assume that the distribution of the outside profit is identical in both populations, i.e. $\Theta_{1}=\Theta_{2}$, which in particular implies $G_{1}=G_{2}:=G$ and $U_{1}=U_{2}$. Therefore, we have $A_{1}=A_{2}=A$. The populations might differ, however, with regard to their infrastructure facilitating spillovers and cost externalities between their members (i.e. with respect to $\beta_{i}$ ).

\section{Symmetric spillovers and slow dynamics}

Discrete time dynamics have the generic property that they might 'overshoot' equilibria if the step size is too large. We do not consider such effects as a mathematical anomaly but as a phenomenon which is observable in many real world markets. However, throughout this first part of the analysis we will avoid this by assuming that the dynamics is "sufficiently slow" (i.e. $\lambda_{1}=\lambda_{2}:=\lambda$ is sufficiently small), such that no local overshooting occurs at any fixed point. This corresponds to a situation where the variance of the outside profit is large. Later on in our analysis we will also deal with the effect of an increase of the speed of the flow in and out of the market.

Let us start with the symmetric case where spillovers in both populations are equal, i.e. $\beta_{1}=\beta_{2}:=\beta$. Initially, we will assume very small spillover effects and characterize how the set of fixed points and the set of initial conditions for the fraction of firms $\left(x_{1,0}\right.$ and $x_{2,0}$ ) which converge to these equilibria change as $\beta$ is increased. In the limit case $\beta=0$, the curves $F_{1}$ and $F_{2}$ are coinciding straight lines defined by $A-B\left(x_{1}+x_{2}\right)-C=0$, which according to assumption (4) is below the line $x_{1}+x_{2}=1$. Note that this implies that we have a continuum of interior fixed points. Generically, in such a case different initial conditions lead to different long run states.

If we slightly increase $\beta$, the curve $F_{1}$ bends upwards; the intersection point with $x_{1}=0$ is fixed and the intersection point with $x_{2}=0$ moves to the right. The curve $F_{2}$ changes in 


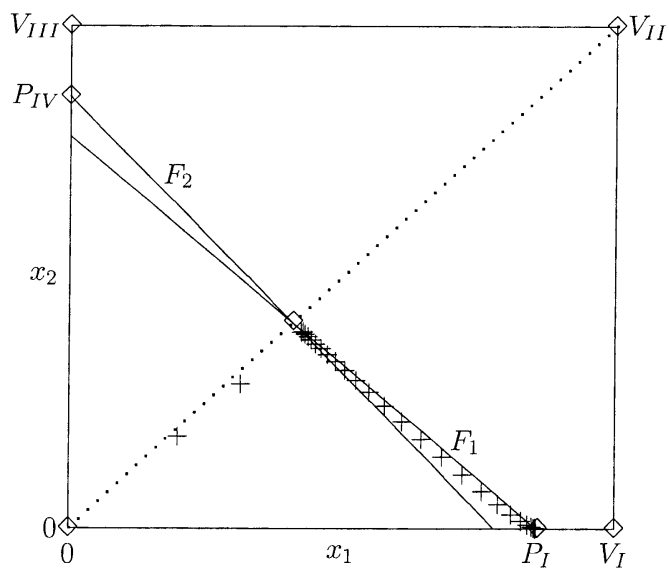

Fig. 1. The curves $F_{i}, i=1,2$ for $\beta<\hat{\beta}$ and a trajectory of the process for $\left(x_{1,0}, x_{2,0}\right)=(0.2,0.18)$ $\left(P_{0}=300, B=100, C=190, U=32, \beta=0.05, \hat{\beta}=0.131\right)$.

a symmetric way. We can therefore conclude that for positive, but very small values of $\beta$, additionally to the four vertices, there are three fixed points: the single interior equilibrium, $S, P_{\mathrm{I}}$ on the line $x_{1}=0$ and $P_{\mathrm{IV}}$ on the line $x_{2}=0$ (see Fig. 1). Due to (4), the local stability conditions (6) and (9) for $V_{\mathrm{I}}$ and $V_{\mathrm{III}}$ are violated for sufficiently small $\beta$. Thus, the two equilibria on the boundary, $P_{\mathrm{I}}=\left(p_{\mathrm{I} 1}, 0\right)$ and $P_{\mathrm{IV}}=\left(0, p_{\mathrm{IV} 2}\right)$, where $p_{\mathrm{I} 1}$ and $p_{\mathrm{IV} 2}$ are identical and satisfy $(A-B p-C) /(1+\beta p)=0$, are the only two stable equilibria. We know that the interior fixed point, $S$, is a saddle point and the complete symmetry of the dynamics with respect to $x_{1}$ and $x_{2}$ implies that the stable set of the saddle is the diagonal of the unit square. Fig. 1 depicts the curves $F_{1}, F_{2}$, all coexisting fixed points and the diagonal. ${ }^{5}$

Due to the symmetry properties, the diagonal is invariant with respect to the dynamics and this suggests, that it separates the basins of attraction of the two stable fixed points $P_{\mathrm{I}}$ and $P_{\mathrm{IV}}$. In order to rigorously establish this fact, we have to show that the dynamics never maps a point from one side of the diagonal to the other side. One way to show this, is to prove that the diagonal is not only forwards, but also backwards invariant with respect to the dynamics. A simple continuity argument then establishes that if there is a backwards invariant curve either all points are mapped from one side to the other or none. Since we know that, for example, $T\left(V_{\mathrm{I}}\right)=V_{\mathrm{I}}$, showing that the diagonal is backwards invariant is sufficient to show that it separates the basins of attraction of $P_{\mathrm{I}}$ and $P_{\mathrm{IV}}$. If the inverse of the generating map of the dynamics is single-valued, it is trivial that the forwards invariant diagonal is also backwards invariant. In order to see whether the inverse is unique, it is useful to consider the so-called critical curves LC of the map $T$. Critical curves separate areas where the number of (rank-1) preimages of points coincide. Whenever points have different numbers of (rank-1) preimages, there has to be at least one critical curve between

\footnotetext{
${ }^{5}$ In all our numerical illustrations we use the parameter values, $P_{0}=300, B=100, C=190, U=32$ for the market environment and expected outside profits. These values satisfy assumptions (3) and (4) for all $\beta_{i} \in[0,1]$. Other values of the parameters which satisfy these assumptions would yield qualitatively similar results.
} 
these points (see Mira et al. (1996), Bischi and Kopel (2001), Bischi et al. (2000) for more details on critical curves). If we denote the set of all points where the determinant of the Jacobian of the map $T$ vanishes by $\mathrm{LC}_{-1}$, then the critical curve LC can be determined by applying the map $T$ to all points of this set, i.e. $\mathrm{LC}=T\left(\mathrm{LC}_{-1}\right)$. In Appendix $\mathrm{B}$, it is shown that every intersection of a critical curve and the diagonal is a critical point of the restriction of $T$ to the diagonal. Accordingly, the number of preimages of $T$ and its restriction to the diagonal coincides for elements of this set. This implies that if the number of rank-1 preimages of points on the diagonal is greater than 1 , all additional rank-1 preimages have to be on the diagonal. Therefore, the diagonal is backwards invariant and indeed separates the basins of attraction of $P_{\mathrm{I}}$ and $P_{\mathrm{IV}}$.

An intuitive interpretation of this result can be given easily. If spillovers are very small and symmetric, the population of firms which initially has the smaller fraction of firms in the market completely leaves the market in the long run and the population with the larger initial market share completely takes over the market. However, since spillovers (and hence cost externalities) are small, it only pays to be in the market if the price is rather high. Consequently, if the number of firms from the own population in the market is too large, using the outside option is, on average, more advantageous, even if the other population has completely left the market. As a consequence, only a certain fraction of firms stays in the market in the long run, whereas, some members of the population (together with all firms of the other population) end up choosing the outside option.

Looking at the transient part of a path where the initial number of firms in the market is small in both populations with slight advantages for population 1, it can be observed that during the early periods the market is attractive for both populations and both $x_{1}$ and $x_{2}$ increase (see Fig. 1). At some point, however, the number of firms in the market becomes so large that firms in population 2 (which enjoy only smaller cost reductions due to spillovers) start leaving the market. Since the market is still attractive for population 1, these firms are replaced by members from population land the path converges to $P_{\mathrm{I}}$. So, the strong effect of the small initial advantages in market share for population 1 becomes apparent only with a certain delay, but in the long run only the industry cluster with the initial advantage in market share will survive.

If the size of spillover effects goes up, the size of the population 1 firm cluster in equilibrium $P_{\mathrm{I}}$ increases until eventually for

$$
\beta=\hat{\beta}:=\frac{C}{A-B}-1>0,
$$

cost reducing externalities are sufficiently large such that producing for the market is profitable for population 1 firms even at a price which arises if the entire population is in the market. Put formally, the fixed point $P_{\mathrm{I}}$ wanders through $V_{\mathrm{I}}$, which becomes locally asymptotically stable 6 . For the same parameter value of $\beta$ also $P_{\mathrm{IV}}$ moves through $V_{\mathrm{III}}$ and $V_{\mathrm{III}}$ becomes locally asymptotically stable. Thus, now the equilibria $(1,0)$ and $(0,1)$ are the

\footnotetext{
${ }^{6}$ Mathematically speaking, we have a transcritical bifurcation (Lorenz, 1993, p. 111). Note that this bifurcation occurs only for $A-B>0$. Otherwise, for all values of $\beta$ only $P_{\mathrm{I}}$ and $P_{\mathrm{IV}}$ are stable. We ignore this rather uninteresting case in the further analysis.
} 


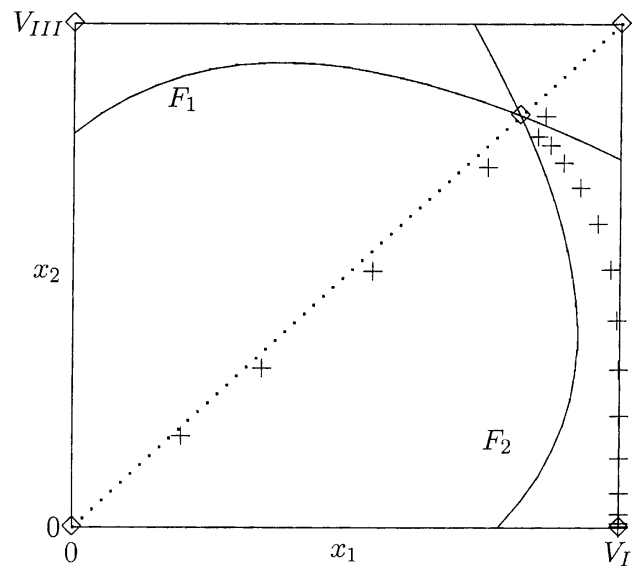

Fig. 2. The curves $F_{i}, i=1,2$ for $\beta \in\left(\hat{\beta}, \beta^{*}\right)$ and a trajectory of the process for $\left(x_{1,0}, x_{2,0}\right)=(0.2,0.18)$ $\left(P_{0}=300, B=100, C=190, U=32, \beta=1, \hat{\beta}=0.131, \beta^{*}=1.209\right)$.

only two stable equilibria. The stable manifold of the interior saddle point still separates the basins of attraction. We depict the equilibria and the corresponding curves $F_{1}, F_{2}$ in Fig. 2 .

We now have a situation where the cost savings in the market due to spillovers are sufficiently large to make the market option always attractive if there are no firms from the other population in the market. However, spillovers are still not large enough to make the market in the long run attractive for the population with the smaller market share. Trajectories here look very similar to the ones observed in Fig. 1. If both groups initially are small, then the number of firms from both populations increases. However, at some point firms from the population with the smaller market share start leaving the market and, eventually, this group vanishes from the market. On the other hand, all firms in the population which initially has the larger market share eventually enter the market. Thus, for such values of the parameters the long run result is a complete market takeover. Since the distribution of the outside profit - which is the profit earned by all firms who left the market-is identical in both populations, it follows that the firms in the population which initially has the the larger fraction of firms in the market end up with higher profits.

The discussion earlier gave us the rather intuitive result that for small $\beta$ increasing local externalities lead to a long-run concentration of the market. However, it turns out that a symmetric increase in local spillovers has quite the opposite implication as $\beta$ becomes large. Considering the transient path of the two cluster sizes makes the argument quite transparent. Assume again that initially both cluster sizes grow. For the relatively small values of $\beta$ considered earlier the in-flow of firms will lower the price to a level, which makes the market unattractive for firms in the smaller cluster, while the larger cluster still grows. Hence, the price keeps going down even as firms in the smaller cluster stop entering and start leaving. However, if spillover effects are sufficiently large such that the price level, where firms in the smaller cluster stop entering, is reached at a time when the entire other population is already in the market, such a stop of entry results in constant prices and (in expectation) a constant size of both clusters. Clearly, the resulting size of the smaller cluster 
is larger the larger the parameter $\beta$ is (i.e. the later this 'indifference price' is reached). Therefore, one has to expect that, after a certain point, further increases in the size of $\beta$ lead to a decrease in the long run concentration of the market.

To analyze this phenomenon more rigorously we observe that whereas the intersection point of $F_{1}$ with the border line $x_{1}=0$ is not affected by the size of $\beta_{1}=\beta$, the remaining part of the curve moves upwards when $\beta$ is increased and eventually touches the horizontal border line $x_{2}=1$. The exact value of $\beta$ where this tangency occurs is given in the following Lemma.

Lemma 1. For $\beta>\beta^{*}$, where $\beta^{*}>0$ is the larger of the two roots of

$$
\left((A-B) \beta^{*}-B\right)^{2}-4 \beta^{*} B(C-(A-B))=0
$$

the curve $F_{1}$ has two positive intersection points with $x_{2}=1$ and $F_{2}$ has two positive intersection points with $x_{1}=1$.

The pair of intersection points of $F_{1}$ and $x_{2}=1$ might either appear left or right of the corner $(1,1)$ of the unit square. In cases where the curve $F_{1}$ touches the line $x_{2}=1$ right of $(1,1)$, the behavior of the system in $[0,1]^{2}$ does not change. Thus, we focus on the case where $F_{1}$ touches $x_{2}=1$ inside the unit square. We will see that in this case the number of locally stable equilibria and the qualitative properties of the process suddenly change. But before we go on to discuss this transition, we summarize the findings for small $\beta$ in the following proposition.

Proposition 2. If spillover effects are symmetric between the two populations with $\beta<\beta^{*}$, all firms from the population with the smaller initial market share eventually leave the market and the market is completely taken over by firms from the population with the larger initial market share. Depending on the size of $\beta$ only a fraction or all of these firms stay in the market in the long run.

For $\beta>\beta^{*}$ four additional fixed points arise which all correspond to mixed market equilibria. Two of them on the boundary $x_{1}=1$ and two of them on $x_{2}=1$. In each of these two pairs only one fixed point is locally stable though. In the following proposition we characterize stability and basins of attraction of all the fixed points under these changed circumstances.

Proposition 3. For $\beta>\beta^{*}$ and sufficiently small $\lambda$ there are four locally stable equilibria$V_{\mathrm{I}}, P_{\mathrm{II}}, V_{\mathrm{III}}, P_{\mathrm{III}}$ - with simply connected basins of attraction. The boundaries of the basins are given by the diagonal and by the stable manifold of $Q_{\mathrm{II}}$ between the basins of $V_{\mathrm{I}}$ and $P_{\mathrm{II}}$ and the stable manifold of $Q_{\mathrm{III}}$ between the basins of $V_{\mathrm{III}}$ and $P_{\mathrm{III}}$.

Thus, we now have four coexisting locally stable equilibria, two with market takeovers in the long run and two where clusters of firms from both populations stay in the market. This makes the characterization of the different basins substantially more difficult than in the cases we have looked at so far. We will illustrate the results concerning basins of attractions by figures depicting the different basins and-primarily in the section on fast 


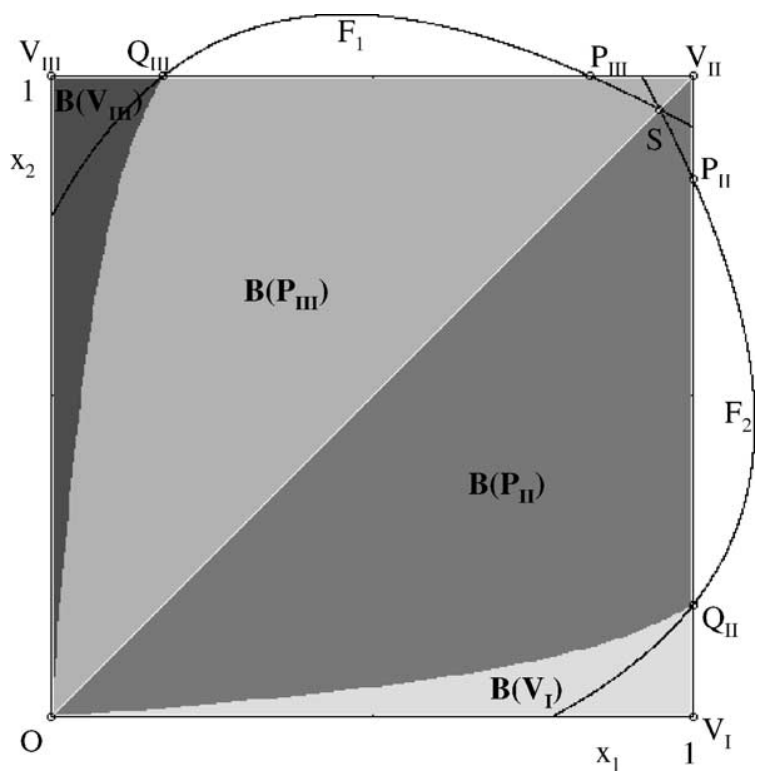

Fig. 3. The basins of attraction of the four stable equilibria for $\beta>\beta^{*}$ and slow dynamics $\left(P_{0}=300\right.$, $B=100, C=190, U=32, \beta=1.5, \beta^{*}=1.209$ ).

dynamics-use the actual shape of the critical curves of the dynamics. This, however, cannot be done without further specification of the switching function $G$. In the numerical illustrations we provide later we always use the specification $G(x)=(2 / \pi) \arctan (\lambda \pi x / 2)$, where $\lambda=\lambda_{1}=\lambda_{2}$. This function satisfies all the assumptions for the switching function $G$ and $\lambda=G^{\prime}(0)^{7}$. In Fig. 3 we use this specifications to depict the basins of attraction and the equal profit curves for symmetric and large spillover effects.

Proposition 3 shows that if spillovers increase symmetrically in both populations-for example because the exchange of information within firms of a population (e.g. a country) is made easier due to improvements in information technology - at some point the long term properties of the system abruptly change. Although it remains true that the population which initially has the larger market share will keep a larger market share also in the long run, for $\beta>\beta^{*}$ it needs quite a large initial advantage to be able to drive firms from the other population completely out of the market. For most initial conditions the system will end up in a state where all firms from the population with the larger initial market share are in the market, but at the same time a large fraction of firms of the other population is able to stay in the market as well (e.g. the equilibrium $P_{\mathrm{III}}$ if $x_{1,0}<x_{2,0}$ ). It is obvious that the market price is much lower at the state $P_{\mathrm{III}}$ than at the state $V_{\mathrm{III}}$. Consequently, the payoff for firms of population 2 is smaller at the boundary equilibrium than at the vertex $V_{\text {III. }}$ On

\footnotetext{
${ }^{7}$ We decided to use this specification rather than, for example, the switching function resulting from a normal distribution for pragmatic reasons: a closed form representation makes the calculation of critical curves easier. With the proper parameterization, a very close match with the switching function stemming from a normal distribution can be achieved, and it is quite obvious that the qualitative properties of the dynamics do not depend on the exact specification.
} 
the other hand, since $P_{\mathrm{III}}$ is an equilibrium where a fraction of the firms in population 1 is in the market and a fraction chooses the outside option, it is obvious that the average profit of a population 1 firm in this equilibrium is identical to the average outside profit. Therefore, the average profit in $P_{\mathrm{III}}$ is identical to the average profit of population 1 firms in $V_{\mathrm{III}}$. If we look at the long run outcomes for initial conditions in the basin of attraction of $P_{\mathrm{III}}$, we can conclude that the difference in profits between firms in the population with the larger and with the smaller initial market share respectively suddenly shrinks if $\beta$ becomes larger than $\beta^{*}$ and the new equilibria appear. Whereas, for $\beta<\beta^{*}$ a symmetric increase of spillover effects in both populations is positive for the long run profit of the firms in the cluster with an initial advantage in market share, the crossing of the threshold $\beta^{*}$ has a negative effect for these firms. Note also that this implies that the equilibria $V_{\mathrm{I}}$ and $V_{\mathrm{III}}$ Pareto dominate the equilibria $P_{\mathrm{II}}$ and $P_{\mathrm{III}}$.

Before we go on to consider asymmetric spillover effects, we like to point out that given our assumptions (3) and (4) no more qualitative changes of the properties of the equilibria or the structure of the basins occur if $\beta$ is further increased.

\section{Asymmetric spillovers and slow dynamics}

Up to now we have only considered a scenario where the cost savings due to spillovers are symmetric in both populations. We have seen that in such cases the population which initially has the larger market share will keep this advantage also in the long run. Additionally, it has been demonstrated how the size of this advantage depends on the value of the spillover parameter $\beta$. Now we will turn to the case where firms from one population operate in an environment which provides superior possibilities for knowledge exchange and information flow. We will analyze how such an advantage modifies the relationship between initial market shares of a cluster and the long run outcome of the evolutionary process with respect to the scenario analyzed earlier.

We start with the symmetric situation depicted in Fig. 2, where spillovers are below $\beta^{*}$ and the two fixed points $V_{\mathrm{I}}$ and $V_{\mathrm{III}}$ are the only attractors. We recall that in this situation for each of the two populations in the long run either all firms are in the market or out of the market depending on the initial market share of the cluster. Now, let us consider a scenario where $\beta_{2}$ stays constant, but for population 1 the conditions of the environment are changed such that the effect of spillovers between firms becomes more significant, i.e. $\beta_{1}$ is increased.

To understand the impact of such an asymmetry on the constellation of equilibria we again refer to the equal profit curves. The curve $F_{i}$ depends only on $\beta_{i}$ but not on $\beta_{j}, j \neq i$ and, therefore, curve $F_{1}$ rotates upwards whereas, curve $F_{2}$ remains unchanged. Initially, the only effect of an increase in $\beta_{1}$ is that the interior saddle point moves up along the unchanged curve $F_{2}$. The basins of attraction of the equilibria $V_{\mathrm{I}}$ and $V_{\mathrm{III}}$ are separated by the stable manifold of the interior fixed point which now lies above the diagonal (note that the diagonal is no longer invariant with asymmetric parameter values). Hence, given that spillovers in population 1 are higher than in population 2, the former is able to take over the market even if the initial number of firms from the other population on this market is slightly larger (Fig. 4a). So, increasing spillover effects in population 1, yields 'continuous' effects on the long run market shares if the increase is only small. Considering the insights 

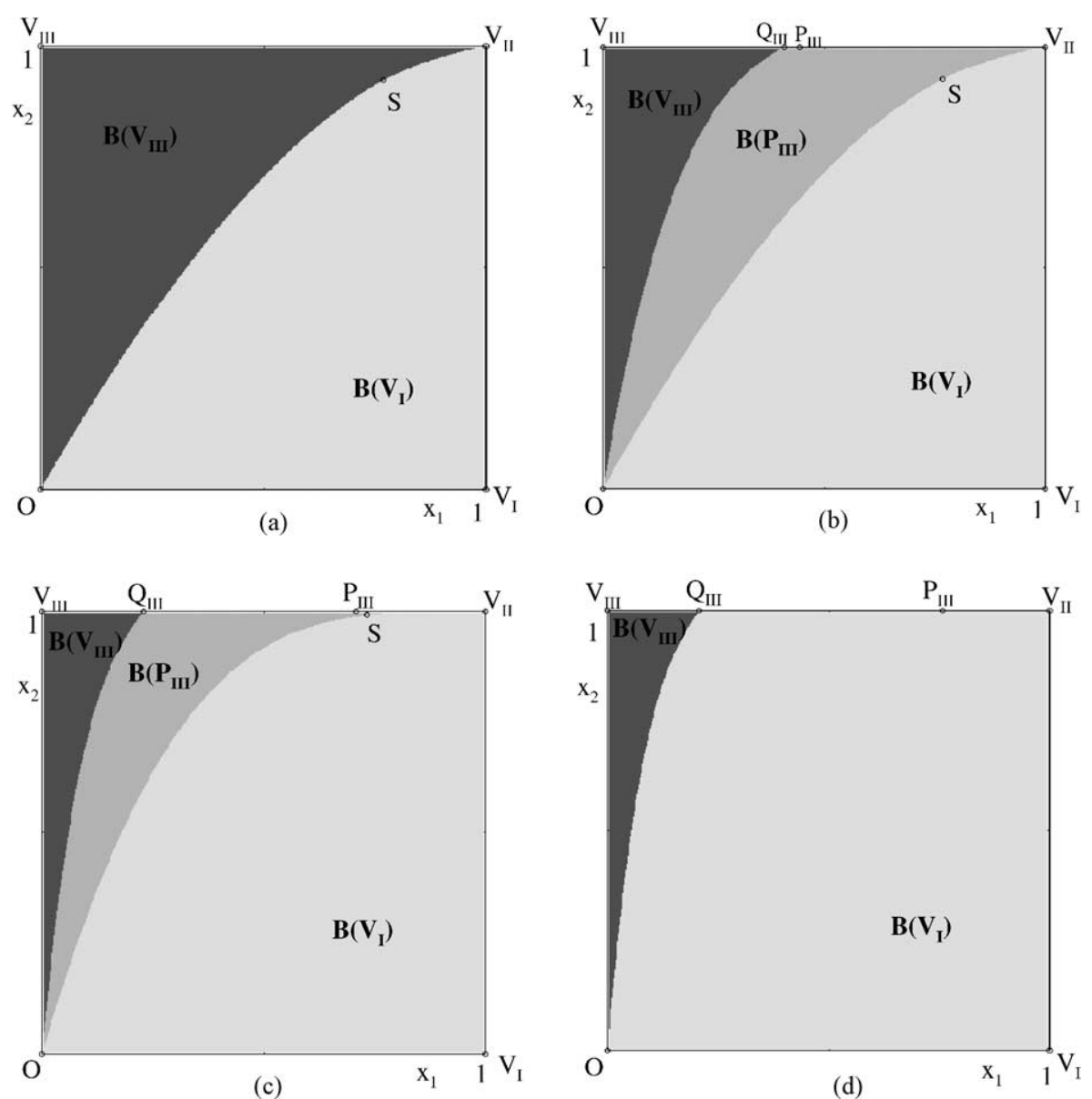

Fig. 4. The basins of attraction of the locally stable equilibria for $\beta_{2} \in\left(\bar{\beta}_{2}, \beta^{*}\right), \beta_{1}>\beta_{2}$, no external spillovers and slow dynamics: (a) $\beta_{1}=1.2<\beta^{*}$; (b) $\beta_{1}=1.21 \in\left(\beta^{*}, \tilde{\beta}_{1}\right)$; (c) $\beta_{1}=1.35 \in\left(\beta^{*}, \tilde{\beta}_{1}\right)$; (d) $\beta_{1}=1.4>\tilde{\beta}_{1}$. $\left(P_{0}=300, B=100, C=190, U=32, \beta_{2}=1, \bar{\beta}_{2}=0.542, \beta^{*}=1.209, \tilde{\beta}_{1}=1.37\right)$.

from the analysis of the case with a symmetric increase of $\beta$, one might however expect that eventually a stable mixed market equilibrium arises where a population 1 cluster coexists with a population 2 cluster of maximum size. It turns out that this only holds true if the spillover effects in population 2 are not too small:

Proposition 4. Assume that $\beta_{2} \in\left(\bar{\beta}_{2}, \beta^{*}\right)$, where

$$
\bar{\beta}_{2}:=\frac{B+C-A+\sqrt{C(B+C-A)}}{A-B} .
$$

(i) For $\beta_{1}>\beta^{*}$ defined in Lemma 1 there appears a pair of fixed points $P_{\mathrm{III}}$ and $Q_{\mathrm{III}}$ on $x_{2}=1$, where $Q_{\mathrm{III}}$ is a saddle point and $P_{\mathrm{III}}$ is locally asymptotically stable. 
For all values $\left(x_{1,0}, x_{2,0}\right)$ between the stable manifolds of $Q_{\mathrm{III}}$ and $S$, in the long run all firms of population 2 and a fraction $p_{\text {IIII } 1}$ of firms in population 1 are in the market.

(ii) As $\beta_{1}$ becomes larger than $\tilde{\beta}_{1}=\left(\beta_{2}\left(1+\beta_{2}\right) B\right) /\left(\left(1+\beta_{2}\right)(A-B)-C\right)>\beta^{*}$, $P_{\mathrm{III}}$ becomes unstable, its basin of attraction suddenly disappears, and for all initial values $\left(x_{1,0}, x_{2,0}\right)$ below the stable manifold of $Q_{\mathrm{III}}$ all firms from population 2 eventually leave the market which is completely taken over by population 1 (convergence to $V_{\mathrm{I}}$ ).

For values of $\beta_{2}$ which are not too small, advantages of population 1 in terms of the size of spillover effects do not have continuous effects on the success of this firm cluster in the market. Rather, it is important to cross the two thresholds $\beta^{*}$ and $\tilde{\beta}_{1}$ to be able to stay in the market or take over the market, even if the other population is initially dominant in the market.

The discontinuous transition of the basins of attraction is somehow similar to the symmetric case. As $\beta_{1}$ crosses $\beta^{*}$, all of a sudden, for all initial conditions between the stable manifold of $Q_{\mathrm{III}}$ and the stable manifold of the interior equilibrium $S$, population 2 no longer controls the whole market in the long run, but a fraction $p_{\mathrm{III} 1}$ of firms of population 1 will stay in the market (Fig. 4b). Now for more than half of all possible initial market conditions population 1 eventually controls the whole market and for an additional set of initial conditions with positive measure, at least a certain fraction of firms from population 1 stays in the market. Note that this huge competitive advantage for population 1 can be gained by a rather small advantage in the parameter $\beta$ (in the example depicted in Fig. $4 \mathrm{~b}$ it is about $20 \%$ ).

If $\beta_{1}$ is further increased, the effect is, for some time, continuous again. The interior equilibrium and, therefore, also its stable manifold (which is the boundary between the basins of attraction of $P_{\mathrm{III}}$ and $V_{\mathrm{I}}$ ) moves up and to the left. Hence, the basin of $V_{\mathrm{I}}$ continuously expands as $\beta_{1}$ is increased. In Fig. $4 \mathrm{c}$ we show the basins of the three coexisting stable equilibria, where the difference in spillovers in the two populations is almost twice as large as in Fig. 4b. We see that the extents of the three basins have not significantly changed. The only noticeable changes are a slight increase in the extent of the basin of $V_{\mathrm{I}}$ and a small reduction of the basin of $V_{\mathrm{III}}$. However, starting from this situation, if $\beta_{1}$ is only slightly increased above $\tilde{\beta}_{1}$, another abrupt structural change in the basins can be observed (Fig. $4 \mathrm{~d}$ ). The basin of attraction of the equilibrium $P_{\text {III }}$ suddenly disappears and is replaced by a part of the basin of attraction of the equilibrium $V_{\mathrm{I}}$. Accordingly, in such a situation for a rather large set of initial conditions, despite the fact that population 2 has a larger initial market share, the cluster in population 1 is not only able to survive, but can eventually take over the entire market and drive all firms from population 2 out.

Even larger advantages of $\beta_{1}$ compared to $\beta_{2}$ expand the basin of $(1,0)$, but we know from our analysis of the symmetric case that the saddle point $Q_{\mathrm{III}}$ always stays to the right of $V_{\mathrm{III}}$. Accordingly, the basin of $(1,0)$ can never cover the whole unit square. In other words, regardless of the size of the difference in the spillovers, there are always initial market conditions such that the population with the smaller spillovers is able to eventually drive firms from the other population out of the market. Of course, the extent of the set containing these initial market conditions becomes very small as the difference in spillover effects becomes large. 
On the other hand, if we start with a small symmetric level of spillovers $\left(\beta_{1}=\beta_{2}<\bar{\beta}_{2}\right)$ and increase the size of spillovers in population 1 , no additional stable equilibria arise and we get an entirely continuous transformation of the basins of attraction:

Proposition 5. Assume that $\beta_{2} \in\left(C /(A-B)-1, \bar{\beta}_{2}\right)$. Then, the only stable equilibria for $\beta_{1}>\beta_{2}$ are $V_{\mathrm{I}}$ and $V_{\mathrm{III}}$. The basin of attraction of $V_{\mathrm{I}}$ always increases continuously in $\beta_{1}$.

The reason that we can never observe mixed market equilibria in such a scenario is that given the relatively small cost externalities in population 2, a population 2 cluster of maximum size can only be profitable if the coexisting cluster of population 1 is small. However, as long as clusters are small, the positive marginal cost effect of one additional firm in the cluster outweighs the negative price effect. Accordingly, if the small population 1 cluster is profitable, it will further grow and eventually drive out the population 2 cluster. If it is not profitable, it will shrink and disappear. In both cases market takeovers can be observed in the long run. This also makes the more general point that a mixed market equilibrium can only be stable if both clusters have a size where the marginal price effects outweighs the marginal cost effect. As we have shown, a certain minimal level of spillover effects is a necessary condition for this to happen.

\section{Asymmetric spillovers and fast dynamics}

Up to now we have assumed that the dynamics of the switching behavior of firms in the two populations is rather slow, i.e. that the parameter $\lambda$ is very small. This means that the probability that a firm changes to the option which is, on average, more attractive is slowly increasing in the difference of the expected profits. Although it might be argued that the increasing synchronization of the entry and exit decisions, which results in a discrete time setup like this if the switching intensity is increased, is not realistic, we still believe that the consideration of such dynamics is relevant. Lags between the point of decision making and the point when the decision is observable in the market lead to scenarios with structures closer to a discrete than a continuous time model. Just considering the recent dynamics of the number of internet-based businesses offers a nice real world example of simultaneous massive entry into a market with subsequent synchronous exits. We will investigate how faster dynamics influence the effect of differences in spillovers. Let us again consider the scenario depicted in Fig. $4 \mathrm{~b}$ where the spillovers in population 1 are larger than the threshold value $\beta^{*}$, whereas $\beta_{2} \in\left(\bar{\beta}_{2}, \beta^{*}\right)$. In this situation three locally stable equilibria coexist. Note that Fig. $4 \mathrm{~b}$ depicts a scenario where switching is slow $(\lambda=0.2 / \pi)$. If the speed of switching is increased $(\lambda=2.2 / \pi)$, the basin boundaries change (Fig. 5a). Although the boundary between the basins of $V_{\mathrm{I}}$ and $P_{\mathrm{III}}$ is no longer smooth, ${ }^{8}$ the change of the basins is continuous in $\lambda_{i}$ and we still have three simply connected basins.

\footnotetext{
8 The non-smoothness of the basin boundary is due to the fact that the interior equilibrium was transformed from a saddle to an unstable node by a sequence of local bifurcations. For a parameter setting like the one in Fig. 5a the boundary is formed by the stable set of a cycle, and the closure of such a stable set also includes many repelling nodes, whose presence yields the non-smoothness of the boundary.
} 

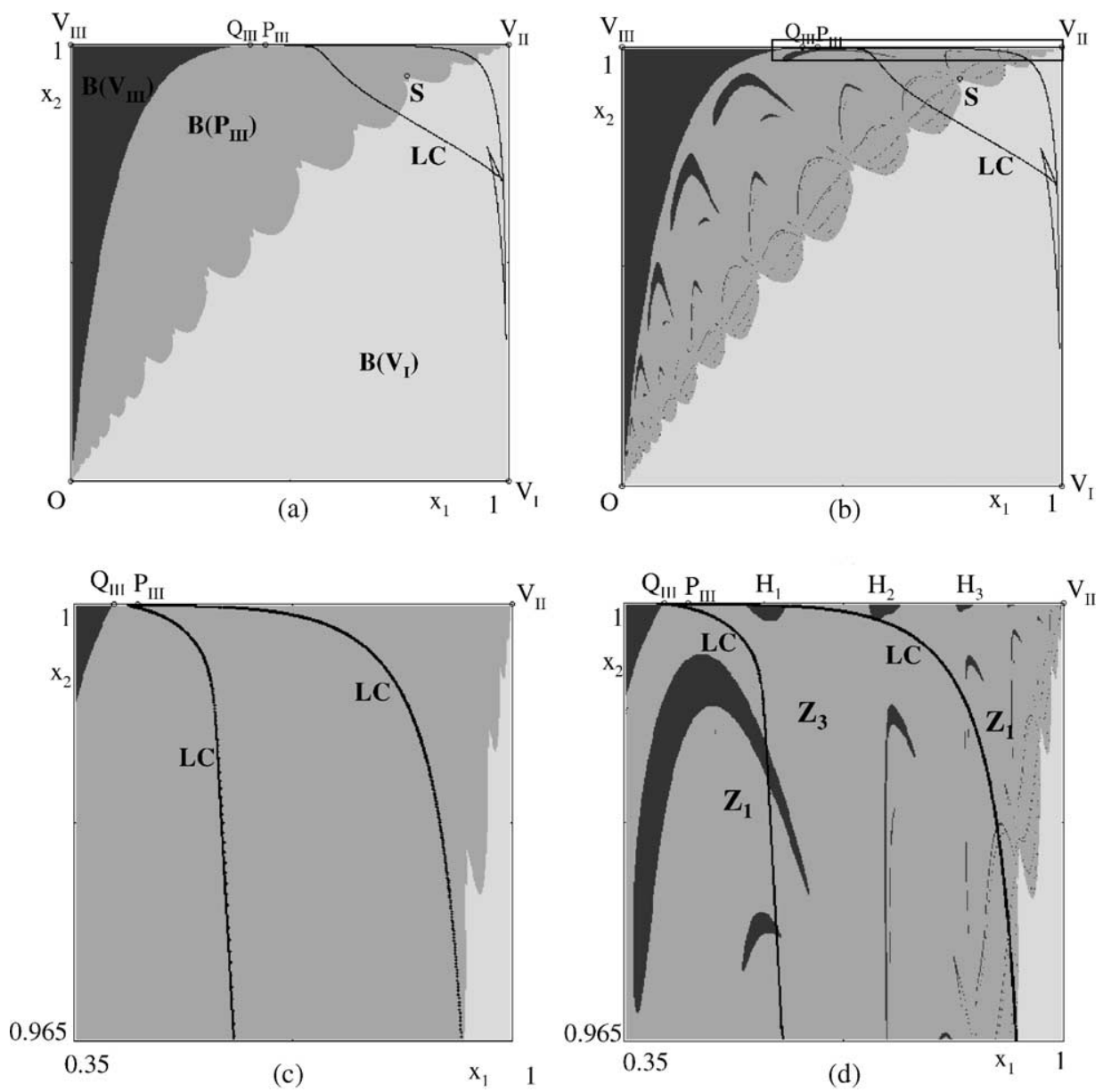

Fig. 5. The transition of the basins of attraction of the three locally stable equilibria as the dynamics becomes faster and the critical curve crosses a basin boundary: (a) no intersection of the critical curve with the boundary between the basins of $V_{\mathrm{III}}$ and $P_{\mathrm{III}}(\lambda=2.2 / \pi)$; (b) the critical curve has crossed the boundary between the basins of $V_{\mathrm{III}}$ and $P_{\mathrm{III}}(\lambda=2.6 / \pi)$; (c) and (d) enlargement of the area where the intersection between LC and the basin boundary occurs. $\left(P_{0}=300, B=100, C=190, U=32, \beta_{1}=1.21, \beta_{2}=1\right)$.

However, if $\lambda$ is increased a little bit further, a quite remarkable change of the basins of attraction can be observed (Fig. 5b). The basin of attraction of $V_{\text {III }}$ has become non-connected, i.e. disjoint portions of it (so-called "islands") are nested inside the basin of another equilibrium. The basin of the boundary equilibrium $P_{\mathrm{III}}$ is now a multiply connected set (i.e. connected with "holes" inside it). This has quite interesting and surprising implications. For a given number of firms in population 1 in the market, an increase in the initial number of firms from population 2 in the market does not necessarily imply a higher long run market share for this population. On the contrary, a higher initial fraction of firms in the market may 
lead to a long run market share of 0 whereas a lower initial fraction leads to convergence to $P_{\mathrm{III}}$ and the long run survival of a firm cluster from population 2 in the market.

The reason for this phenomenon lies in the interaction of overshooting effects at the equilibrium $P_{\mathrm{III}}$ with the cost externalities imposed by the spillovers. Given the fast switching behavior of firms, convergence to the mixed market equilibrium $P_{\mathrm{III}}$ is no longer monotonic, but the size of the population 1 cluster fluctuates due to excessive entry and exit of firms in this population. Of course this results also in price fluctuations but these fluctuations are dampening and in the absence of externalities the process would end up at $P_{\mathrm{III}}$. However, in the presence of spillover effects there are global implications of the overshooting behavior at $P_{\mathrm{III}}$. If the number of population 1 firms in the market in period $t$ is slightly larger than

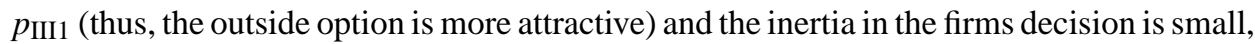
so many firms leave the cluster in population 1 that afterwards the size of the spillovers falls short of the threshold making this cluster viable. Accordingly, the remaining firms leave the market in the following periods and the cluster vanishes although a cluster of size $p_{\text {III1 }}$ would have been viable. Initially, it is virtually impossible to predict whether the fluctuation will lead the cluster size below the threshold and this creates the complex basins of attraction.

Note that the constellation of fixed points and their local stability properties have not changed in this transition to faster dynamics. Accordingly, and this is important to realize, local analysis cannot be used to explain this change in the long run properties of the process. In the remainder of this section we demonstrate how the theory of critical curves can be employed to analyze such a global bifurcation from a mathematical point of view. Although the complex basins are in our case created by local overshooting effects, this technique can be used for the examination of the structure of the basins of attraction of any non-invertible dynamics.

In Fig. $5 \mathrm{c}$ it can be observed that both $\mathrm{LC}_{-1}$ (which is the locus where the determinant of the Jacobian vanishes, det DT $\left.\left(x_{1}, x_{2}\right)=0\right)$ and $\mathrm{LC}=T\left(\mathrm{LC}_{-1}\right)$ are closed curves. The region outside $\mathrm{LC}$ is the region $Z_{1}$ of points with only one rank-1 preimage, and inside $\mathrm{LC}$ there are points with three rank-1 preimages, i.e. the region $Z_{3}$. Note that the region $Z_{3}$ is entirely included in the basins of $P_{\mathrm{III}}$ and $V_{\mathrm{I}}$ for this value of $\lambda$. As $\lambda$ is increased, the critical curve LC and the stable set of the boundary fixed point $Q_{\text {III }}$, which constitutes the boundary between the basins of $P_{\mathrm{III}}$ and $V_{\mathrm{III}}$, have a contact (in fact, numerical evidence reveals that the first contact of LC and the boundary which separates the basins occurs along the boundary $x_{2}=1$ ). After this contact occurred, a small portion of $Z_{3}$ enters the basin of $V_{\text {III }}$ (compare Fig. $5 \mathrm{c}$ and d). This means that suddenly a small portion of the basin of $V_{\text {III }}$ has a larger number of preimages, namely three instead of one. The two new rank-1 preimages of this portion merge along $\mathrm{LC}_{-1}$ (Fig. $5 \mathrm{~d}$ ). Since they are inside $Z_{3}$ these preimages again have three (rank-1) preimages (which are rank-2 preimages of the small region which has been created when LC crossed the basin boundary). This leads to an arborescent sequence of preimages. All these preimages belong to the basin of attraction of $V_{\text {III }}$, since they are mapped into the immediate basin of $V_{\text {III }}$ after a finite number of iterations.

Since the first contact of LC and the basin boundary between $P_{\mathrm{III}}$ and $V_{\mathrm{III}}$ occurs along $x_{2}=1$, the occurrence of the global bifurcation which changes the structure of the basins can be understood by looking at the one-dimensional restriction of the map $T$ to the invariant 


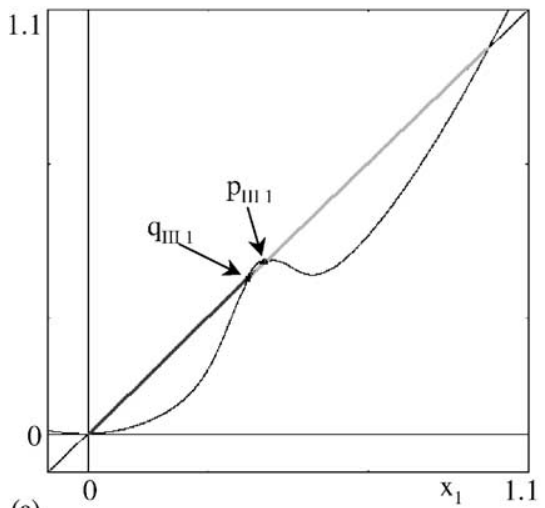

(a)

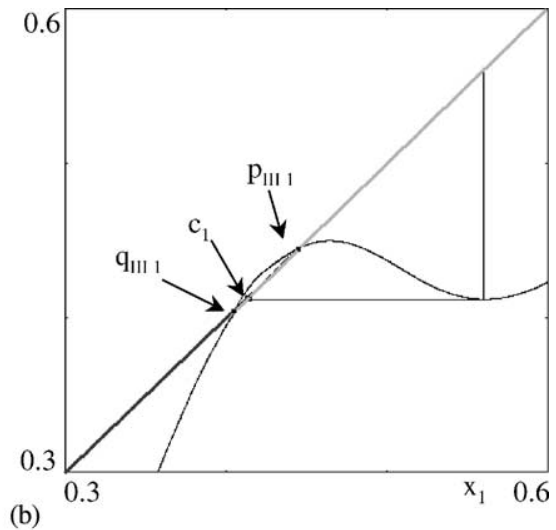

(b)

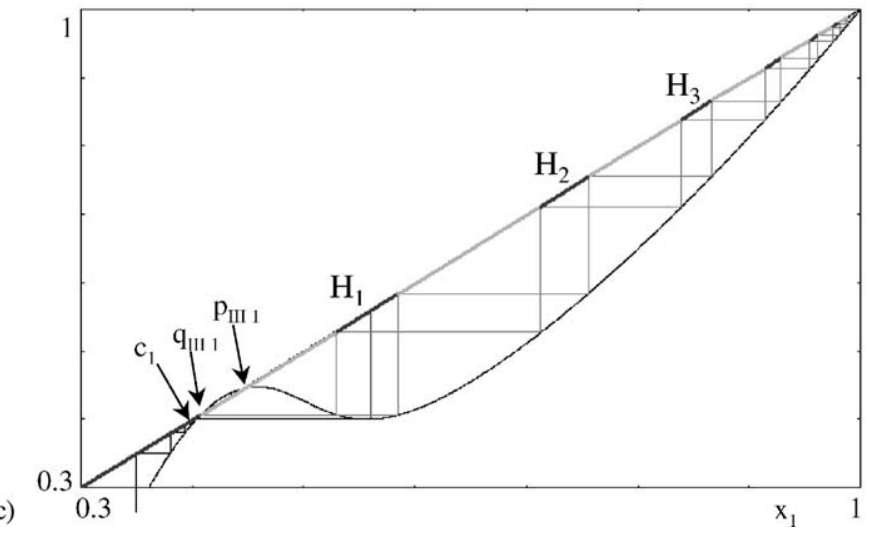

Fig. 6. Illustration of the effect of the crossing of a basin boundary by a critical curve for the one-dimensional restriction of $T$ to the line $x_{2}=1$ : (a) and (b) $\lambda=2.2 / \pi$; (c) $\lambda=2.6 / \pi$. $\left(P_{0}=300, B=100, C=190, U=32\right.$, $\left.\beta_{1}=1.21\right)$.

line $x_{2}=1 .{ }^{9}$ The critical points (local maxima and minima) of these restrictions are the intersections of LC and $x_{2}=1$. In Fig. 6 the graphs of this restriction, $h(x)$ see (A.6) is shown for the parameter values corresponding to Fig. $5 \mathrm{a}$ and $\mathrm{b}$ respectively.

In Fig. 6a the two stable fixed points have connected basins bounded by unstable fixed points. The local minimum is inside the basin of the positive stable fixed point (see the closeup in Fig. 6b). The change of $\lambda$ first causes a contact of the local minimum and the unstable fixed point. After this contact a "hole" of the basin of $x=0$ is created around the minimum. This can be clearly seen in Fig. 6c, where $H_{1}, H_{2}$ and $H_{3}$ indicate points right of $p_{\text {IIII } 1}$ which are mapped to the left of $q_{\mathrm{III} 1}$ in 1,2 respectively 3 iterations. All the points in these intervals, therefore, belong to the basin of attraction of 0 . It should be pointed out that such a 'basin bifurcation' has to occur for any switching function $G$, where $\lambda$ is

\footnotetext{
${ }^{9}$ It should be emphasized that this is a special characteristic of this model, and no general property of this kind of basin bifurcations.
} 
sufficiently large and $\beta$ sufficiently close to $\beta^{*}$. So, the transition which is responsible for non-connected basins in this framework does not depend on the exact specification of $G$.

As $\lambda$ is further increased, the portion of the immediate basin of $V_{\mathrm{III}}$ inside $Z_{3}$ becomes larger and, consequently, the holes enlarge leading to even more fragmented and intermingled basins. It is evident that although the attractors continue to be simple equilibria (stable fixed points) the structure of the basins is getting more and more complex. This causes a greater uncertainty about the long-run evolution of the system starting from a given initial condition in the following sense: A small change in the starting condition has a high probability to cause a crossing of a basin boundary and, as a consequence, the convergence to a different equilibrium. Hence, the long run outcome of the process now depends very sensitively on the initial number of firms from both populations in the market.

We summarize the findings of this global analysis in our final proposition.

Proposition 6. For $\beta_{2} \in\left(\bar{\beta}_{2}, \beta^{*}\right)$ and $\beta_{1}>\beta^{*}$, where $\beta_{1}$ is sufficiently close to $\beta^{*}$ and $\lambda$ sufficiently large, the basin of attraction of $V_{\mathrm{III}}$ is non-connected and has islands in the basin of attraction of $P_{\mathrm{III}}$. The number of population 2 firms which stay in the market in the long run does not increase monotonously in the initial number of population 2 firms in the market.

\section{Discussion}

In this paper we have used an evolutionary industry model to study the effect of spillovers on the development of competing firm clusters. The decision of a firm whether to produce a good for the market or to choose some outside option is made using a simple imitation type decision rule. We have focused our analysis on the long run outcome of the dynamic adaptation process in both populations and in particular have characterized the basins of attraction of the stable market constellations for different parameter values. We have demonstrated that a dynamic analysis of the evolution of market shares yields very precise insights into the relationship between the size of spillover effects, the inertia in the process of market exit and entry, initial market shares and the long run success of a firm cluster in the market. The main findings of this analysis may be summarized as follows:

- For symmetric spillovers initial advantages in market share lead to long-run dominance in the market. For growing industries this takeover occurs with a delay after an initial growth period for both clusters.

- Increasing symmetric spillover effects increases market concentration if current spillovers are small but decreases market concentration if current spillovers are large. In any case, it facilitates the growth of the initially smaller firm cluster. The set of initial conditions where the smaller cluster stays in the market increases with the size of the spillovers.

- If levels of spillovers are sufficiently large in both populations, increasing differences in spillovers have discontinuous effects on the basins of long run equilibria. The set of initial sizes where a cluster stays in the market increases abruptly as the advantages in the spillovers crosses certain thresholds.

- For fast exit and entry behavior the sets of initial market shares yielding long run dominance of different populations may be intermingled. Hence, long run market shares may 
depend sensitively on initial market shares and are not always monotonously increasing in the initial cluster size. The faster the switching behavior the larger is the set of initial conditions where the population with the smaller spillover effects can take over the market.

More generally, we have shown that the analysis of dynamic evolutionary models with non-linear payoff structure may be challenging due to the presence of numerous coexisting locally stable equilibria with complicated basins of attraction. For a sound understanding of the long run properties of the process, a characterization of these basins is necessary and we have demonstrated how the theory of critical curves can be used in addition to local bifurcation theory to explain the structural changes in the long run properties of the process. The use of this technique should facilitate the applicability of deterministic evolutionary analysis in many other models as well, and could in some cases generate predictions for a more realistic time horizon than a stochastic approach. Thus, we believe that the analysis provided here is of general interest for researchers working in the field of evolutionary modeling as well.

\section{Acknowledgements}

We thank Laura Gardini for helpful comments and suggestions. This work has been performed under the under the auspices of CNR, Italy, and under the activity of the national research project "Nonlinear Dynamics and Stochastic Models in Economics and Finance", MURST, Italy.

\section{Appendix A}

\section{A.1. Proof of Proposition 1}

We start the proof by giving a characterization of the equal profit curves $F_{1}$ and $F_{2}$. The curve of equal profit $F_{1}$ is given by

$$
B \beta_{1} x_{1}^{2}+B \beta_{1} x_{1} x_{2}+\left(B-A_{1} \beta_{1}\right) x_{1}+B x_{2}+C_{1}-A_{1}=0,
$$

and the curve $F_{2}$ satisfies

$$
B \beta_{2} x_{2}^{2}+B \beta_{2} x_{1} x_{2}+\left(B-A_{2} \beta_{2}\right) x_{2}+B x_{1}+C_{2}-A_{2}=0
$$

Thus, these curves are hyperbolae with centers $K_{1}=\left(-1 / \beta_{1},\left(B+A_{1} \beta_{1}\right) / B \beta_{1}\right)$ for $F_{1}$ and $K_{2}=\left(\left(B+A_{2} \beta_{2}\right) / B \beta_{2},-1 / \beta_{2}\right)$ for $F_{2}$. The slopes of the asymptotes are -1 and $-\infty$ for $F_{1}$ and -1 and 0 for $F_{2}$. We concentrate on the properties of $F_{1}$, the corresponding properties of $F_{2}$ follow by symmetry. Since the center of $F_{1}$ is left of $[0,1]^{2}$, the curve $F_{1}$ in this area is upward bending (it has positive curvature). Analogously, the curve $F_{2}$ is downward bending (it has negative curvature). It is further easy to see from (A.1) that there has to be exactly one intersection of $F_{1}$ with the line segment $\{0\} \times[0, \infty)$. Note further that along any straight line in $[0,1]^{2}$ with slope-1 the overall number of firms in the market and, therefore, also the market price stays constant. Since we assume $\beta_{i}>0$, the profit difference $\pi_{i}\left(x_{1}, x_{2}\right)-U_{i}$ increases along any such line. In particular, this means that if we draw a straight line $L$ with 
slope -1 through an arbitrary point $\left(x_{1}, x_{2}\right)$ of $F_{1}$ we have $\pi_{1}\left(\tilde{x}_{1}, \tilde{x}_{2}\right)>U_{1}$ for every point $\left(\tilde{x}_{1}, \tilde{x}_{2}\right) \in L$, such that $\tilde{x}_{1}>x_{1}$ and $\pi_{1}\left(\tilde{x}_{1}, \tilde{x}_{2}\right)<U_{1}$ for every point $\left(\tilde{x}_{1}, \tilde{x}_{2}\right) \in L$, such that $\tilde{x}_{1}<x_{1}$. The same argument shows that if $L$ is a straight line with slope-1 through a point $\left(x_{1}, x_{2}\right)$ on $F_{2} \pi_{2}\left(\tilde{x}_{1}, \tilde{x}_{2}\right)<U_{2}$ for every point $\left(\tilde{x}_{1}, \tilde{x}_{2}\right) \in L$, such that $\tilde{x}_{1}>x_{1}$, and $\pi_{2}\left(\tilde{x}_{1}, \tilde{x}_{2}\right)>U_{2}$ for every point $\left(\tilde{x}_{1}, \tilde{x}_{2}\right) \in L$, such that $\tilde{x}_{1}<x_{1}$.

We will now characterize the fixed points on the boundary. To minimize notation let us denote the boundary line of $[0,1]^{2}$ with $x_{2}=0$ by $\mathrm{BL}_{1}$ the one with $x_{1}=1$ by $\mathrm{BL}_{2}$, the one with $x_{2}=1$ by $\mathrm{BL}_{3}$ and the one with $x_{1}=0$ by BL 4 . Due to our assumptions (3) and (4) there has to be an odd number of intersections of $F_{1}$ respectively $F_{2}$ with $\mathrm{BL}_{1} \cup \mathrm{BL}_{2}$ and an odd number of intersections in $\mathrm{BL}_{3} \cup \mathrm{BL}_{4}$. Note further that due to our assumption that $\beta_{i}>0, F_{1}$ cannot intersect with $\mathrm{BL}_{1}$ without intersecting with $\mathrm{BL}_{4}$ and $F_{2}$ cannot intersect with $\mathrm{BL}_{4}$ without intersecting with $\mathrm{BL}_{1}$. Furthermore, the arguments in the last paragraph imply that if $F_{1}$ intersects with $\mathrm{BL}_{1}$ the whole curve must lie below the straight line with slope-1 through this intersection point, which in particular shows that $F_{1}$ cannot also intersect with $\mathrm{BL}_{3}$. This leaves us with five different cases: (a) The equal profit curve never enters $[0,1]^{2}$. (b) $F_{1}$ has one intersection point with $\mathrm{BL}_{1}$, then there has to be exactly one intersection point with $\mathrm{BL}_{4}$ but no intersection points with $\mathrm{BL}_{2}$ and $\mathrm{BL}_{3}$. If $F_{1}$ has no intersection point with $\mathrm{BL}_{1}$ then there has to be exactly one intersection point with $\mathrm{BL}_{2}$ (if there were more than one intersection points there would have to be at least three which is ruled out by the hyperbolic shape of $F_{1}$ ). This gives three more cases. (c) There is one intersection point with $\mathrm{BL}_{2}$ and one with $\mathrm{BL}_{4}$. (d) There is one intersection point with $\mathrm{BL}_{2}$ and one with $\mathrm{BL}_{3}$; e) there is one intersection point with $\mathrm{BL}_{2}$, two intersection points with $\mathrm{BL}_{3}$ and one intersection point with $\mathrm{BL}_{4}$. The claim of the proposition concerning the boundary equilibria follows directly.

To show that there can be at most one interior fixed point, we simply have to observe that if we draw a line with slope -1 through an intersection point $\left(x_{1}^{*}, x_{2}^{*}\right)$ of $F_{1}$ and $F_{2}$ all points on $F_{1}$ with $x_{1}>x_{1}^{*}$ have to lie above this line, all points on $F_{1}$ with $x_{1}<x_{1}^{*}$ have to lie below this line. On the other hand, every point on $F_{2}$ with $x_{2}>x_{2}^{*}$ has to lie below the line and any point on $F_{2}$ with $x_{2}<x_{2}^{*}$ has to lie above this line. Therefore, there cannot be a second point of intersection of $F_{1}$ and $F_{2}$.

Finally, we show that the interior equilibrium is always unstable. Since we know that the dynamics along the straight line with slope -1 points away from the equilibrium, obviously the interior equilibrium always has to have at least one unstable manifold. Furthermore, it is easy to realize that a straight line between $(0,0)$ and the equilibrium never intersects either $F_{1}$ or $F_{2}$. Given our assumptions about the direction of the dynamics at $(0,0)$ it follows that the dynamics points towards the interior fixed point along this line. Accordingly, the interior fixed point either has to be a saddle point or a repelling node with one positive and one negative eigenvalue, where both have absolute values larger than 1 .

\section{A.2. Proof of Lemma 1}

The condition for $F_{1}$ to have a tangency with $x_{2}=1$ is that the equation

$$
A-B\left(1+x_{1}\right)-\frac{C}{1+\beta x_{1}}=0
$$


which is equivalent to the quadratic equation

$$
\beta B x_{1}^{2}-((A-B) \beta-B) x_{1}-(A-B-C)=0
$$

has a positive real solution. Assumption (4) guarantees that the left hand side is positive for $x_{1}=0$. Hence, if there exist real roots, either both of them are negative or both of them are positive. Real roots exist if

$$
((A-B) \beta-B)^{2}-4 \beta B(C-(A-B)) \geq 0 .
$$

To show that there exists a pair of positive real numbers where the inequality is binding, we observe that the left hand side goes to infinity for $\beta \rightarrow \infty$, is positive for $\beta=0$, and, given assumption (4) is negative for $\beta=B /(A-B)$. We denote the two real roots of (A.5) by $0<\hat{\beta}<\beta^{*}$. It is now straight forward to see that (A.4) has two negative roots for $\beta<\hat{\beta}$, no real roots for $\hat{\beta}<\beta<\beta^{*}$ and two positive roots for $\beta>\beta^{*}$.

\section{A.3. Proof of Proposition 3}

To understand the changes in the number of fixed points and their local and global stability properties as the size of the spillovers cross the level $\beta=\beta^{*}$, it is useful to consider the restriction of the map $T$ to the invariant line $x_{2}=1$. Many properties of the two-dimensional dynamical system on $[0,1]^{2}$ can be inferred from this one-dimensional restriction. For $x_{2}=$ 1 , the time evolution of $x_{1}$ is given by the system $x_{1, t+1}=h\left(x_{1, t}\right)$, where

$$
h\left(x_{1, t}\right)=x_{1, t}+x_{1, t}\left(1-x_{1, t}\right) G\left(A-B\left(x_{1, t}+1\right)-\frac{C}{1+\beta x_{1, t}}\right)
$$

It follows from (4) and continuity that for small values of $\beta$ the graph of $h$ lies below the diagonal on the entire interval $(0,1)$. This implies that the map $h$ has only two fixed points: 0 is locally stable and 1 is unstable. If $\beta$ is increased, at the value $\beta^{*}$ the graph of $h$ touches the diagonal from below and, for even larger $\beta$, a pair of additional fixed points appears ${ }^{10}$ (as pointed out in the text we only consider parameter values where this pair is in $[0,1])$. Clearly, the stability properties of the corner fixed points remain unchanged, whereas the left of the two new additional fixed points, $q_{\mathrm{III} 1}$, is unstable and the right, $p_{\mathrm{III} 1}$ is stable. This can be easily seen in Fig. 7, where we show a schematic representation of the restricted map $h$ for $\beta>\beta^{*}$.

The map $h$ is monotone if $\lambda$ is sufficiently small and thus any trajectory starting right of $q_{\mathrm{III} 1}$ stays right of $q_{\mathrm{III} 1}$ and converges to $p_{\mathrm{III} 1}$. On the other hand, any trajectory with initial condition $x_{1,0}<q_{\mathrm{III} 1}$ converges to 0 . The local stability properties of the additional fixed points $q_{\mathrm{III} 1}$ and $p_{\mathrm{III} 1}$ of $h$ determine the stability of the fixed points $Q_{\mathrm{III}}$ and $P_{\mathrm{III}}$ of the map $T$ along the invariant manifold $x_{2}=1$. Standard local stability analysis shows that the eigenvalue for the transversal eigenvector is given by $1-G\left(\pi_{2}\left(x_{1}, 1\right)-U\right)$, where $x_{1}=q_{\mathrm{III} 1}$ or $x_{1}=p_{\mathrm{III} 1}$ depending on the fixed point we consider. We treat the case of $Q_{\mathrm{III}}$, but all arguments also apply to $P_{\mathrm{III}}$. Since $Q_{\mathrm{III}}$ is a fixed point, we have

$$
\pi_{1}\left(q_{\mathrm{III} 1}, 1\right)-U=A-B\left(1+q_{\mathrm{III} 1}\right)-\frac{1}{1+\beta q_{\mathrm{III} 1}}=0
$$

\footnotetext{
${ }^{10}$ In mathematical terms, the map $h$ undergoes a tangent bifurcation (Lorenz, 1993).
} 


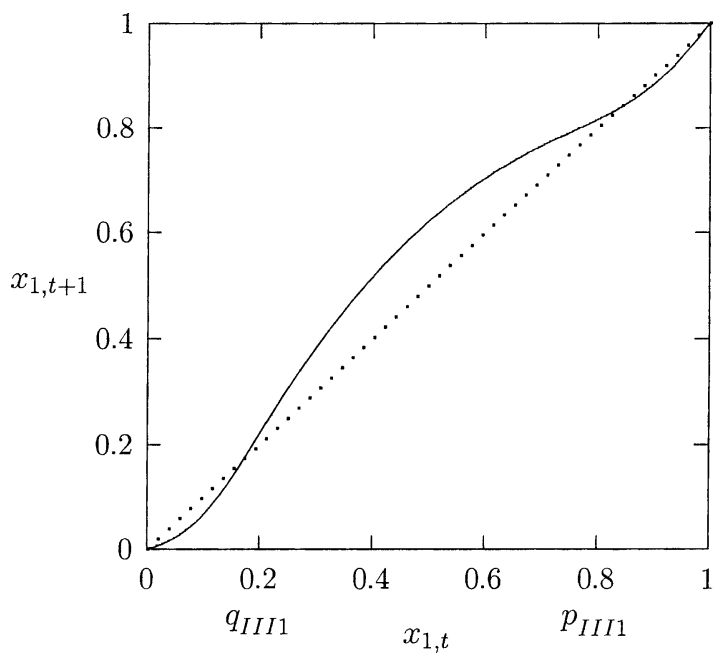

Fig. 7. The restriction of the map $T$ to the line $x_{2}=1$ for $\beta>\beta^{*}$ and slow dynamics $\left(P_{0}=300, B=100\right.$, $\left.C=190, U=32, \beta=1.5, \beta^{*}=1.21\right)$.

which implies

$$
\pi_{2}\left(q_{\mathrm{III} 1}, 1\right)-U=A-B\left(1+q_{\mathrm{III} 1}\right)-\frac{1}{1+\beta}>0 .
$$

Thus, $G\left(\pi_{2}\left(q_{\mathrm{III} 1}, 1\right)-U\right)>0$ and the transversal eigenvalue is in $(0,1)$. This implies that $Q_{\mathrm{III}}$ is a saddle point and $P_{\mathrm{III}}$ is locally asymptotically stable. The same line of arguments as used to show that the backwards invariant diagonal cannot be crossed by a trajectory implies that the stable set of the saddle point $Q_{\text {III }}$ cannot be crossed if all points in this set have only one rank-1 preimage. The properties of $G$ (in particular its S-shape) imply that the map of the dynamics (5) is invertible on $[0,1]^{2}$ for sufficiently small $\lambda$. This implies that the considerations for $x_{2}=1$ can be extended to the whole unit square and for $\beta>\beta^{*}$ and sufficiently small $\lambda$ the stable set of the saddle point $Q_{\mathrm{III}}$ is a smooth curve connecting $Q_{\text {III }}$ and 0 . The stable set of $Q_{\text {III }}$ splits the former basin of attraction of $V_{\text {III }}$ into a smaller basin of $V_{\text {III }}$ and a basin of attraction of the new stable equilibrium $P_{\mathrm{III}}$. The triangular basin below the diagonal undergoes exactly the same transition.

\section{A.4. Proof of Proposition 4}

(i) Like in the symmetric case, if $\beta_{1}$ crosses the threshold value $\beta^{*}$, the curve $F_{1}$ has a contact with the line $x_{2}=1$ and, if $\beta_{1}$ is further increased, a pair of new equilibria $Q_{\text {III }}$ and $P_{\mathrm{III}}$ appears. In contrast to the case of a symmetric increase of $\beta_{1}$ and $\beta_{2}$, this pair of additional equilibria might in general also appear to the right of the intersection of the curve $F_{2}$ with $x_{2}=1$. Hence, we have to show that under condition (12) the point where the curve 
$F_{1}$ touches $x_{2}=1$ for $\beta_{1}=\beta^{*}$ is left to the intersection of $F_{2}$ and $x_{2}=1$. This intersection $\left(\tilde{x}_{1}, 1\right)$ is defined by the equality $A-B\left(1+x_{1}\right)-C /\left(1+\beta_{2}\right)=0$, and, therefore, we have

$$
\tilde{x}_{1}=\frac{1}{B}\left(A-B-\frac{C}{1+\beta_{2}}\right) \text {. }
$$

(ii) If $\beta_{1}$ is increased above $\beta^{*}$, either $Q_{\mathrm{III}}$ or $P_{\mathrm{III}}$ eventually collides with $\left(\tilde{x}_{1}, 1\right)$. To prove our claim we have to show that $P_{\mathrm{III}}$, which is right of $Q_{\mathrm{III}}$, collides with $\left(\tilde{x}_{1}, 1\right)$. Both at $Q_{\text {III }}$ and $P_{\text {III }}$ we have $A-B\left(1+x_{1}\right)-C /\left(1+\beta_{1} x_{1}\right)=0$ and thus when one of the two points collides with $\left(\tilde{x}_{1}, 1\right)$ we must have $\beta_{1} \tilde{x}_{1}=\beta_{2}$, which implies

$$
\beta_{1}=\tilde{\beta}_{1}:=\frac{\beta_{2}\left(1+\beta_{2}\right) B}{(A-B)\left(1+\beta_{2}\right)-C} .
$$

Note that the denominator is positive for $\beta_{2}>\bar{\beta}_{2}$. Considering the derivative of population 1 payoffs with respect to $x_{1}$ at this point for $\beta_{1}=\tilde{\beta}_{1}$, we get

$$
\begin{aligned}
\frac{\partial \pi_{1}\left(\tilde{x}_{1}, 1\right)}{\partial x_{1}} & =-B+\frac{C \tilde{\beta}_{1}}{\left(1+\tilde{\beta}_{1} \tilde{x}_{1}\right)^{2}}=-B+\frac{C \tilde{\beta}_{1}}{\left(1+\beta_{2}\right)^{2}} \\
& =-B+\frac{C B \beta_{2}}{\left(1+\beta_{2}\right)\left((A-B)\left(1+\beta_{2}\right)-C\right)}
\end{aligned}
$$

The last expression is negative if and only if $C \beta_{2} /\left(\left(1+\beta_{2}\right)\left((A-B)\left(1+\beta_{2}\right)-C\right)\right)<1$. Straightforward calculations show that this is true if and only if (12) holds. It follows from $\pi_{1}(1,1)<0$ that, the inequality $\partial \pi_{1}\left(x_{1}, 1\right) / \partial x_{1}<0$ can only hold at $P_{\text {III }}$. Hence, under condition (12) the pair of equilibria appears to the left of the intersection of $F_{2}$ with $x_{2}=1$ and by the same arguments as used in the symmetric case the fixed point $Q_{\mathrm{III}}$ is a saddle point, $P_{\mathrm{III}}$ is locally stable and the basins of $V_{\mathrm{I}}$ and $P_{\mathrm{III}}$ are separated by the stable manifold of $Q_{\mathrm{III}}$.

To show (ii) we only have to observe that for the value $\tilde{\beta}_{1}$ where $\left(\tilde{x}_{1}, 1\right)$ and $P_{\mathrm{III}}$ collide the equal profit curves $F_{1}$ and $F_{2}$ intersect on the line $x_{2}=1$. Accordingly, at this point the interior equilibrium $S$ moves through the equilibrium $P_{\mathrm{III}}$ on the boundary and an exchange of stability occurs. The former stable equilibrium $P_{\mathrm{III}}$ becomes a saddle point and the interior saddle point $S$ leaves the unit square. The stable set of the saddle point $P_{\mathrm{III}}$ is the invariant boundary $x_{2}=1$, but this line now is repelling in the transversal direction in a neighborhood of $P_{\mathrm{III}}$.

\section{A.5. Proof of Proposition 5}

It follows from the proof of Proposition 5 that for $\beta_{2}<\bar{\beta}_{2}$ the pair of new equilibria $Q_{\mathrm{III}}, P_{\mathrm{III}}$ which appears for $\beta_{1}=\beta^{*}$ is initially right of the intersection of $F_{2}$ with $x_{2}=1$. This means that both are unstable in the direction transversal to $x_{2}=1$. Accordingly, $Q_{\mathrm{III}}$ is unstable and $P_{\mathrm{III}}$ is a saddle point where the stable manifold is the line $x_{2}=1$. Thus, also for $\beta_{1}>\beta^{*}$ there exist only two stable equilibria, $V_{\mathrm{I}}, V_{\mathrm{III}}$ and the basin of attraction of $V_{\mathrm{I}}$ increases continuously with increasing $\beta_{1}$, where the basin boundary is still given by the stable manifold of $S$. For $\beta_{1}=\tilde{\beta}_{1}$, the interior equilibrium $S$ wanders through $Q_{\mathrm{III}}$ and the stable manifold of $S$ becomes the stable manifold of $Q_{\text {III }}$ which is now a saddle point. 
Again, no discontinuous changes in the basins of attraction occur and for $\beta_{1}>\tilde{\beta}_{1}$ the basin boundary is the stable manifold of $Q_{\text {IIII }}$.

\section{Appendix B}

Since we consider a case with symmetric parameter constellations we have $T_{1}(x, y)=$ $T_{2}(y, x) \forall(x, y) \in[0,1]^{2}$. Let us denote the restriction of the map to the diagonal by $f(x)$ : $f(x):=T_{1}(x, x)=T_{2}(x, x)$. Thus, $f^{\prime}(x)=T_{1 x_{1}}(x, x)+T_{1 x_{2}}(x, x)$. We show that any intersection of the critical curve separating a region with three preimages, $Z_{3}$, from the region with only one preimage, $Z_{1}$, with the diagonal has to be a critical point of the map $f$. This implies that the number of preimages of $f$ on the diagonal switches from 1 to 3 . Let us denote this intersection by $(z, z)$. Any point on the critical curve has two rank-1 preimages, two merging in a point of $\mathrm{LC}_{-1}$ and an extra preimage. Since the diagonal is invariant, at least one of the two rank-1 preimages of $(z, z)$ has to be on the diagonal. However, since the dynamical system is perfectly symmetric with respect to the diagonal this implies that also the second rank-1 preimage is on the diagonal. At the point $(v, v) \in L C_{-1}$, where the two merging rank-1preimages are located, the determinant of the Jacobian has to vanish. Due to the symmetry of the map $T$ we have $T_{1 x_{1}}(v, v)=T_{2 x_{2}}(v, v), T_{1 x_{2}}(v, v)=T_{2 x_{1}}(v, v)$ and the characteristic polynomial of the Jacobian at $(v, v)$ is given by $\left(T_{1 x_{1}}-v\right)^{2}-T_{1 x_{2}}^{2}$. Thus, the eigenvalues of the Jacobian at $(v, v)$ are given by $v_{1}=T_{1 x_{1}}(v, v)+T_{1 x_{2}}(v, v)$ and $v_{2}=T_{1 x_{1}}(v, v)-T_{1 x_{2}}(v, v)$. Simple calculations show that

$$
\begin{aligned}
T_{1 x_{1}}(v, v)-T_{1 x_{2}}(v, v)= & 1+(1-2 v) G\left(A-2 B v-\frac{C}{1+\beta v}\right) \\
& +v(1-v) G^{\prime}\left(A-2 B v-\frac{C}{1+\beta v}\right) \frac{C \beta}{(1+\beta v)^{2}} .
\end{aligned}
$$

From our assumption that $\beta>0$ it follows from that this expression is positive. Since the Jacobian has to vanish at $(v, v), T_{1 x_{1}}(v, v)+T_{1 x_{2}}(v, v)=0$ has to hold. We know that $f^{\prime}(v)=T_{1 x_{1}}(v)+T_{1 x_{2}}(v)$ and this implies that $z$ has to be a critical point of $f$. Accordingly, the number of additional preimages of points on the diagonal across the critical curve with respect to $T$ coincide with the number of additional rank-1 preimages with respect to $f$. In other words, all additional rank-1 preimages have to be on the diagonal. Observing that the point $(0,0)$ has one rank-1 preimage with respect to $T$ and 0 has one rank-1 preimage with respect to $f$ now establishes that for all points on the diagonal the number of preimages with respect to $T$ and with respect to $f$ coincide. Therefore, the diagonal is for all symmetric parameter constellation forward and backward invariant with respect to $T$.

Having done this we show that for sufficiently fast switching there is local overshooting around the interior fixed point on the diagonal, which implies that there is a region $Z_{3}$ around $S$ in the unit square where $T$ has three preimages. The dynamics along the invariant diagonal reads

$$
x_{t+1}=f\left(x_{t}\right):=x_{t}+x_{t}\left(1-x_{t}\right) G\left(A-2 B x_{t}-\frac{C}{1+\beta x_{t}}\right),
$$


where $x_{1, t}=x_{2, t}=x_{t}$. The derivative of $f$ is given by

$$
\begin{aligned}
f^{\prime}(x)= & +(1-2 x) G\left(A-2 B x-\frac{C}{1+\beta x}\right) \\
& +x(1-x) G^{\prime}\left(A-2 B x-\frac{C}{1+\beta x}\right)\left(-2 B+\frac{\beta C}{(1+\beta x)^{2}}\right) .
\end{aligned}
$$

We have $f(0)=0, f(1)=1$ and, due to assumption (3), $f^{\prime}(0)>1$. Furthermore, we know that there is at most one interior fixed point of the map. If such an interior fixed point $s$ exists, we therefore always have $f^{\prime}(s)<1$. Since the second term in the expression for $f^{\prime}$ has to be 0 at $s$, the third one has to be negative which implies $-2 B+\beta C /(1+\beta s)^{2}<0$. Accordingly, we have

$$
f^{\prime}(s)=1+G^{\prime}(0)\left(-2 B+\frac{\beta C}{(1+\beta v)^{2}}\right),
$$

which is negative for sufficiently large $G^{\prime}(0)=\lambda$. At the value of $\lambda$ where $f^{\prime}(s)=0$, a critical curve appears which surrounds the interior fixed point for larger values of $\lambda$.

\section{References}

Audretsch, D.B., Feldman, M.P., 1996. R\&D Spillovers and the geography of innovation and production. American Economic Review 86, 630-640.

Benabou, R., 1993. Workings of a city: location, education, and production. Quarterly Journal of Economics 108, 619-652.

Bischi, G.I., Kopel, M., 2001. Equilibrium selection in a nonlinear duopoly game with adaptive expectations. Journal of Economic Behavior and Organization 46, 73-100.

Bischi, G.I., Gardini, L., Kopel, M., 2000. Analysis of global bifurcations in a market share attraction model. Journal of Economic Dynamics and Control 24, 855-879.

Carlisle, E.R., 1992. Spillover asymmetries and a comparative technological advantage. The American Economist $36,13-17$.

Chuang, Y.-C., Lin, C.-M., 1999. Foreign direct investment, R\&D and spillover efficiency: evidence from Taiwan's manufacturing firms. Journal of Development Studies 35, 117-137.

Dawid, H., 1999a. On the convergence of genetic learning in a double auction market. Journal of Economic Dynamics and Control 23, 1545-1567.

Dawid, H., 1999b. On the dynamics of word of mouth learning with and without anticipations. Annals of Operations Research 89, 273-295.

Ellison, G., 2000. Basins of attraction, long run stochastic stability and the speed of step-by-step evolution. Review of Economic Studies 67, 17-45.

Ellison, G., Fudenberg, D., 1993. Rules of thumb for social learning. Journal of Political Economy 101, $612-643$.

Ellison, G., Fudenberg, D., 1995. Word-of-mouth communication and social learning. Quarterly Journal of Economics CX, 93-125.

Ellison, G., Glaeser, E.L., 1997. Geographic Concentration in US Manufacturing Industries: A Dartboard Approach. Journal of Political Economy 105, 889-927.

Ellingsen, T., 1997. The evolution of bargaining behavior. Quarterly Journal of Economics. 581-602.

Friedman, D., 1991. Evolutionary games in economics. Econometrica 59, 637-666.

Friedman, D., 1998. On economic applications of evolutionary game theory. Journal of Evolutionary Economics $8,15-43$.

Friedman, D., Fung, K.C., 1996. International trade and the internal organization of firms: an evolutionary approach. Journal of International Economics 41, 113-137. 
Ghemawat, P., Spence, A.M., 1985. Learning curve spillovers and market performance. Quarterly Journal of Economics 100, 839-852.

Kamien, M.I., Muller, E., Zang, I., 1992. Research joint ventures and R\&D, cartels. American Economic Review $82,1293-1306$.

Lorenz, H.-W., 1993. Nonlinear Dynamical Economics and Chaotic Motion, 2d Edition. Springer, Berlin.

Lu, X., McAfee, R.P., 1996. The evolutionary stability of auctions over bargaining. Games and Economic Behavior $15,228-254$.

Mansfield, E., 1988. The speed and cost of industrial innovation in Japan and the United States: external vs. internal technology. Management Science 34, 1157-1168.

Mira, C., Gardini, L., Barugola, A., Cathala, J.-C., 1996. Chaotic Dynamics in Two-Dimensional Noninvertible Maps. World Scientific, Singapore.

Porter, M.E., 1998. On Competition. Harvard University Press, Cambridge, MA.

Qin, C.-Z., Stuart, C., 1997. Are Cournot and Bertrand equilibria evolutionary stable strategies? Journal of Evolutionary Economics 7, $41-47$.

Vega-Redondo, F., 1997. The Evolution of Walrasian Behavior. Econometrica 65, 375-384.

Weibull, J.W., 1995. Evolutionary Game Theory. MIT Press, Cambridge, MA.

Young, P., 1993. An evolutionary model of bargaining. Journal of Economic Theory 59, 145-168.

Young, P., 1998. Individual Strategy and Social Structure: An Evolutionary Theory of Institutions. Princeton University Press, Princeton. 